Production, Manufacturing and Logistics

\title{
A continuous review replenishment-disposal policy for an inventory system with autonomous supply and fixed disposal costs
}

\author{
Çerağ Pinçe ${ }^{a}$, Ülkü Gürler ${ }^{b, *}$, Emre Berk ${ }^{c}$ \\ a Erasmus Research Institute of Management, Erasmus University, Rotterdam, The Netherlands \\ ${ }^{\mathrm{b}}$ Department of Industrial Engineering, Bilkent University, Ankara, Turkey \\ ${ }^{\mathrm{c}}$ Faculty of Business Administration, Bilkent University, Ankara, Turkey
}

Received 5 June 2004; accepted 5 April 2007

Available online 30 June 2007

\begin{abstract}
In this study, we analyze an inventory system facing stochastic external demands and an autonomous supply (independent return flow) in the presence of fixed disposal costs and positive lead times under a continuous review replenishmentdisposal policy. We derive the analytical expressions of the operating characteristics of the system; and, construct the objective function to minimize the total expected costs of ordering, holding, purchasing and disposal per unit time subject to a fill rate constraint. An extensive numerical analysis is conducted to study the sensitivity of the policy parameters and the benefit of employing a policy which allows for disposal of excess stock in this setting. We model the net demand process as the superposition of normally distributed external demand and inflows, which is expressed as a Brownian motion process. Our findings indicate that the disposal option results in considerable savings even (i) in the presence of non-zero fixed disposal costs, (ii) large actual demand rates with high return ratios (resulting in small net demands) and (iii) for moderate return ratios with high demand variability.
\end{abstract}

(C) 2007 Elsevier B.V. All rights reserved.

Keywords: Inventory control; Reusables; Disposal; Brownian motion; Autonomous supply

\section{Introduction}

In this paper, we study a single item, single-location inventory system facing continuous stochastic demand with two sources of supply. One source is an external supplier with a constant lead time and the other is an independent continuous autonomous supply (inflow) joining stock immediately upon arrival. Replenishment from the outside supplier incurs variable and fixed costs. Due to the stochastic nature of the inflow process, inventories may reach undesirably high levels and it may be desirable to dispose of the excess stock in the form

\footnotetext{
* Corresponding author. Tel.: +90 312 2902413; fax: +90 3122664054.

E-mail address: ulku@bilkent.edu.tr (Ü. Gürler).
} 
of sales in a secondary market and/or temporary relocation. We assume that both replenishment and disposal actions incur variable and non-zero fixed costs. Zero setup cost for disposal (zero fixed disposal cost) makes perfect sense when the system can operate as a gatekeeper, especially for big items, such as, home appliances and used cars. However, for other types of goods (i.e. returnable containers, refillable bottles), there may be non-zero fixed costs associated with disposing of excess stock and/or stopping the inflow of reusable goods. The main contribution of our work lies in that we take into account settings, where disposals may incur non-zero fixed costs.

An example that the authors are familiar with comes from refillable bottles used in the beverage industry in Denmark, where all beer producers are required to use refillable bottles and to be registered with the Dansk Retursystem, a non-profit organization coordinating the deposit-return activities (see www.dansk-retursystem.dk). Danish beer producers are responsible by law for the recollection of refillable bottles and for refunding of deposit on return plus the logistic fee to Dansk Retursystem. Although there is an effective depositreturn system bearing high return rates (98-99\%), demand for new bottles is inevitable due to depreciation and loss of refillables. Therefore, when on-hand refillable bottles are not enough to satisfy the production demand, new bottles are ordered from an outside supplier. Both kinds of bottles are deemed immediately usable. Inherent demand variability combined with take-back obligations may result in excess stocks of returned bottles at certain times which requires disposal or relocation. For example, the management at Tuborg, a large Danish brewer, has the option of relocating some of the bottle stocks at the Fredericia brewery to another of its warehouses near Copenhagen, if holding them at the former location is deemed not desirable [3]. Such a relocation action may involve some fixed as well as variable costs of transportation. Use of identical bottles by different firms creates also the opportunity of exchanging excess bottles in a secondary market for some firms. Similar inventory systems exist in bottle-washing companies providing new and resterilized glass bottles mostly to wine producers (CWC and Skookum Inc. [4]). (We refer the reader to Platt and Rowe [17] and González-Torre et al. [13] for other examples of similar practice.)

Another example of this setting is returnable containers that are used as secondary packaging materials (e.g., pallets, drums and slipsheets). Such standardized containers are interchangeable among different users and are immediately reusable after inspection and cleaning operations of negligible duration (e.g., IBM, Ford, General Motors and Toyota using returnable containers instead of one-way packaging materials as told in $[24,1,16])$. Typically, a third-party logistic (3PL) service provider manages the reusable containers for a number of industrial customers. The inventory system in this study mimics a depot for such a 3PL organization. At the aggregate level, the return and demand processes are independent and stochastic. Strict safety and fire hazard regulations exist for the storage of such containers; and, at some locations, there are physical capacity limits that inventory levels cannot exceed (e.g. a pile volume of wooden pallets cannot exceed 100,000 cubic feet in the state of California [City of Newark Fire Prevention Bureau [5]]). Kroon and Vrijens [16] observed that stock is relocated when there is an imbalance in the amount of containers held at different depots. Such relocation actions may be viewed as temporary disposals and may involve some fixed as well as variable costs of transportation. A European network of logistics firms using identical pallets also creates opportunities in secondary markets to exchange excess inventory (see Europal, www.europal.net).

Inventory management of such reusable containers and packaging materials has recently become more important due to the increased governmental regulations on take-back obligations for consumer protection and recycling/waste reduction objectives for environmental protection (see e.g. the European Parliament and Council Directive 94/62/EC, [10] and Platt and Rowe [17]). Beside such regulatory pressures, companies have also found it economically beneficial to cultivate a 'green' image among the consumers (see [23]).

There have been a number of studies in the literature on inventory management of reusables. In the brief literature review below, we mainly focus on the continuous review models.

The earliest work for an inventory system facing stochastic demands and returns is Heyman [15] who examines a single-item inventory system with unit Poisson processes and the options of purchase, repair and discarding of excess stock. In an extension, he also provides a diffusion approximation based on the simple netting of demand and return processes. Both models assume zero lead times and negligible fixed costs for the replenishment, repair and disposal options. Van der Laan et al. [20] consider the disposal option for the above model in the presence of a positive lead time with zero fixed and non-zero variable costs. In [22], van der Laan and Salomon consider a single-product, hybrid production/inventory system where push-dis- 
posal and pull-disposal strategies are employed. The disposal action incurs only positive variable costs. For the same control strategies, the effects of lead time characteristics on total costs are investigated by van der Laan et al. [21]. Teunter and Vlachos [19] study a single-item production system with manufacturing and remanufacturing under periodic review in the presence of disposal options. They assume that there is no setup cost for disposal (fixed disposal cost is zero). Using simulation, they numerically show that disposal is an economically viable option if an item is slow-moving, the recovery rate is high and remanufacturing is as expensive as manufacturing.

To the best of our knowledge, the only works that consider non-zero fixed disposal costs are Constantinides [6], Constantinides and Richard [7], Beltran and Krass [2], and Golany et al. [12]. The first two consider stochastic demands with zero lead times and analyze the optimal two-sided $(s, S)$ control policy. The last two consider a deterministic demand and return setting in the presence of disposal options with concave costs and provide polynomial solution algorithms.

We are not aware of any study in the literature that models a stochastic inventory system with an autonomous supply in addition to a regular supplier when (i) there is a positive lead time for delivery from the regular supplier and (ii) there are non-zero fixed (setup) costs for disposal. In this paper, we provide this model for constant lead times, normally distributed demands and autonomous supply. Since disposals incur positive setup, we employ a four-parameter replenishment-disposal control policy under continuous review. Our main contribution is that we model explicitly the non-zero fixed disposal cost. Thus, our analytical model enables one to determine the optimal disposal batch size and timing (in terms of inventory levels) as well as the order size and the reorder point that minimize the expected cost rate in the presence of non-zero fixed replenishment and disposal costs. Our numerical study also supports the previous findings with zero fixed disposal costs that disposals may result in considerable savings even when disposals incur non-zero setup costs.

The rest of this paper is organized as follows: in Section 2, we introduce the basic assumptions of the inventory model and the control policy. In Section 3, we provide some preliminary results for the Brownian motion process, and in Section 4, the operating characteristics of the system are derived. In Section 5, we provide the results of our numerical study on the sensitivity of the policy parameters to various system parameters and on the benefits of incorporating a disposal option. Finally, in Section 6, we present our concluding remarks.

\section{The model}

We consider a single item, single-location inventory system facing continuous stochastic demand. Inventory holding cost is charged at $h$ per unit of on-hand stock per unit time. All unmet demand is backordered under a desired fill rate measure. The system has two external sources of supply. One is a regular supplier with ample capacity and has a positive shipment lead time, $L$. The other supply source is a stochastic, continuous autonomous supply which joins the stock and may be used to satisfy the demand immediately. As such, we do not model remanufacturing or recovery operations explicitly; similarly, we consider yields on these operations outside the scope of our analysis. We assume that the inflow of material is beyond managerial control except for a batch disposal option. That is, we exclude regulation of the autonomous supply process through economic or non-economic mechanisms, but solely rely on discarding accumulated stock. External material inflows are not sufficient to satisfy the entire demand due to depreciation and loss. Hence, the stock is occasionally replenished from an outside regular supplier with a fixed cost of $K_{\mathrm{o}}$ for each replenishment order and a unit purchasing cost of $c_{\mathrm{o}}$ for every unit. Furthermore, a positive cost $c_{r}$ is incurred per unit of autonomous supply.

The inflow per unit time, $X_{\mathrm{R}}$, and external demand per unit time, $X_{\mathrm{D}}$, are assumed to be stationary normal random variables with means $\mu_{\mathrm{R}}$ and $\mu_{\mathrm{D}}$, and variances $\sigma_{\mathrm{R}}^{2}$ and $\sigma_{\mathrm{D}}^{2}$, respectively. We define the net demand per unit time, $X_{\mathrm{N}}=X_{\mathrm{R}}-X_{\mathrm{D}}$. Then, $X_{\mathrm{N}}$ is normal with mean $\mu=\mu_{\mathrm{R}}-\mu_{\mathrm{D}}$ and variance $\sigma^{2}=\sigma_{\mathrm{R}}^{2}+\sigma_{\mathrm{D}}^{2}-$ $2 \operatorname{Cov}\left(X_{\mathrm{R}}, X_{\mathrm{D}}\right)$ where $\operatorname{Cov}\left(X_{\mathrm{R}}, X_{\mathrm{D}}\right)$ is the covariance of inflow and demand per unit time. Both the inflows from the autonomous supply and demands are assumed to be independent over non-overlapping unit time intervals. Consequently (in conjunction with the stationarity and normality assumptions), the cumulative net demand at time $t$ is expressed as a Brownian motion process with drift $\mu$ and variance coefficient $\sigma^{2}$. In a realistic setting with more demand than returns on average, $\mu$ may take zero or negative values only. Simplistic netting of inflows and external demands via a single parameter distribution has been found to be inadequate to capture the true behavior due to negligence of added variances (e.g. Poisson process). Excellent 
discussions on the inadequacy of single parameter netting approaches can be found in $[20,9,11]$. The use of a two-parameter distribution herein overcomes this netting difficulty.

If the inflow and external demand processes are Brownian motion processes, this net flow is an exact representation. If the processes themselves are not Brownian motions, for high rates of inflows and external demands of, say, Poisson processes, Brownian motion assumption can be taken as a good continuous approximation (see [15]). Due to the existence of an autonomous supply process, on-hand inventories may accumulate to levels which are deemed prohibitively high from either an economic perspective (e.g., excessive holding costs) or a physical perspective (e.g., storage capacity limitations). In such cases, it may be desirable to dispose of some of the accumulated stock. We consider positive fixed and variable costs of disposal, $K_{\mathrm{d}}$ and $c_{\mathrm{d}}$, respectively. The objective is to minimize the expected total cost rate subject to a fill rate constraint. The expected total cost consists of expected ordering, holding, purchasing and disposal costs; backordering costs are treated implicitly. We express the fill rate measure through the fraction of time that there is positive inventory on hand; we will set the fill rate measure to be at least $\alpha$ where $0<\alpha<1$.

With both disposal and reordering options, we employ the following four-parameter continuous review policy:

Policy: A replenishment order of size $Q$ is placed whenever the inventory position drops to the reorder point $r$, and, whenever the inventory position exceeds the disposal trigger level $S$, excess inventory is disposed of to bring the inventory position down to $s$ immediately, so long as there are no outstanding orders.

This control policy will be referred to as the $(S, s, r, Q)$ policy, where $Q$ and $r$ denote the replenishment decision variables, and $S$ and $s$ denote the disposal decision variables. A continuous review control policy may be implemented when the inventory system can be monitored continuously at negligible cost as the modern technology currently enables; and, it provides a lower bound on the expected cost rate with respect to its periodic review counterpart. We consider only non-negative values for the control variables in our analysis and set $S \geqslant s$ and $s>r$. The first follows from the definition of a disposal trigger level and the second is required since, otherwise, a disposal would trigger a simultaneous reordering decision.

For analytical convenience, we assume that the inventory level is always above the reorder point $r$ when an order is received. That is, we assume that the net demand during a lead time period never exceeds the ordering quantity. With this assumption, we, slightly, overestimate holding and disposal costs and, underestimate ordering and purchasing costs, fill rate and time between successive replenishment orders. Since, under the proposed policy, disposal and reordering decisions are made only when there are no outstanding orders, there will be only one outstanding order at any time.

In our analysis, we use the renewal theoretic approach. Under the control policy stated above, the stochastic process representing the inventory position repeats itself probabilistically at the epochs when the inventory position hits $r$. Therefore, the reordering instances constitute regeneration points and a cycle is defined as the time between two consecutive order placements. A cycle consists of two segments - a lead time period of constant length, $L$, and a stochastic segment lasting from the receipt of the order until the next reorder instance. The derivation of the expressions for the operating characteristics of the inventory system rests on some fundamental results regarding the behavior of Brownian motions moving either in an unrestricted fashion or within a strip (an interval bounded from above and under). We present these results in the next section and proceed with the derivation of the operating characteristics in Section 4.

\section{Preliminaries}

In this section we present some results on the behavior of a Brownian motion moving within a strip. From Cox and Miller [8, p. 222], we immediately have

Proposition 3.1. Let $b<x<a$. Then, for a Brownian motion $\{X(t), t \geqslant 0\}$ with drift $\mu$, variance coefficient $\sigma^{2}$ and $X(0)=x$, moving in the strip $[b, a]$, the probability density function of the location, $y$, of $X(t)$ is given by

$$
f_{X(t)}(y, t, x)=\mathrm{e}^{\frac{\mu(y-x)}{\sigma^{2}}} \sum_{n=1}^{\infty} a_{n} \mathrm{e}^{-\lambda_{n} t} \sin \left(\frac{n \pi(y-b)}{a-b}\right),
$$

where 


$$
a_{n}=\frac{2}{a-b} \sin \left(\frac{n \pi(x-b)}{a-b}\right) \lambda_{n}=\frac{1}{2}\left[\frac{\mu^{2}}{\sigma^{2}}+\left(\frac{n \pi \sigma}{a-b}\right)^{2}\right] .
$$

Next, we consider the probability of a Brownian motion hitting the boundaries of a strip and the corresponding expected first escape times. Although their derivation rest on known properties, some of them are not readily available in the literature. For this, we introduce the following variables: $T_{u, v}$ denotes the time the Brownian motion, starting at $u$, hits $v$ for the first time; and, $T_{u, v}^{z}$ denotes the time it takes the process, starting at $u$, to hit $v$ for the first time, without hitting $z$, where $z<u<v$. Finally, $T_{u, v z}$ denotes the time it takes the process, starting at $u$, to escape from the strip $[v, z]$ for the first time. Similarly, let $P_{u, v}^{z}$ be the probability that the process, starting at $u$, hits $v$ before $z$, that is $P_{u, v}^{z}=P\left(T_{u, v}<T_{u, z}\right)$.

Proposition 3.2. Let $b<x<a$. Then for a Brownian motion $\{X(t), t \geqslant 0\}$ with $X(0)=x$, drift $\mu$, variance coefficient $\sigma^{2}$ and moving in the strip $[b, a]$, we have

(i) $P_{x, a}^{b}=\frac{\left(\mathrm{e}^{-\frac{2 \mu x}{\sigma^{2}}}-\mathrm{e}^{-\frac{2 \mu b}{\sigma^{2}}}\right)}{\left(\mathrm{e}^{-\frac{2 \mu a}{\sigma^{2}}}-\mathrm{e}^{-\frac{2 \mu b}{\sigma^{2}}}\right)} \quad$ and $P_{x, b}^{a}=\frac{\left(\mathrm{e}^{-\frac{2 \mu a}{\sigma^{2}}}-\mathrm{e}^{-\frac{2 \mu x}{\sigma^{2}}}\right)}{\left(\mathrm{e}^{-\frac{2 \mu a}{\sigma^{2}}}-\mathrm{e}^{-\frac{2 \mu b}{\sigma^{2}}}\right)}$,

(ii) $E\left(T_{x, b a}\right)=-\frac{x}{\mu}+\frac{(a-b)}{\mu\left(\mathrm{e}^{-\frac{2 \mu a}{\sigma^{2}}}-\mathrm{e}^{-\frac{2 \mu b}{\sigma^{2}}}\right)} \mathrm{e}^{-\frac{2 \mu x}{\sigma^{2}}}+\frac{b \mathrm{e}^{-\frac{2 \mu a}{\sigma^{2}}}-a \mathrm{e}^{-\frac{2 \mu b}{\sigma^{2}}}}{\mu\left(\mathrm{e}^{-\frac{2 \mu a}{\sigma^{2}}}-\mathrm{e}^{-\frac{2 \mu b}{\sigma^{2}}}\right)}$.

Proof. See Appendix.

For the special case of $\mu=0$, Proposition 3.2 reduces to

Corollary 3.1. Let $b<x<a$. Then for a Brownian motion $\{X(t), t \geqslant 0\}$ with $X(0)=x, \mu=0$, variance coefficient $\sigma^{2}$ and moving in the strip $[b, a]$, we have

(i) $P_{x, a}^{b}=\frac{x-b}{a-b} \quad$ and $\quad P_{x, b}^{a}=\frac{a-x}{a-b}$,

(ii) $E\left(T_{x, b a}\right)=\frac{(x-b)(a-x)}{\sigma^{2}}$.

Finally, we need following results for the expected on-hand derivations.

Proposition 3.3. Let $b<x<a$. Then for a Brownian motion $\{X(t), t \geqslant 0\}$ with $X(0)=x$, drift $\mu$, variance coefficient $\sigma^{2}$ and moving in the strip $[b, a]$, the expected area under its trajectory until the escape time is

$$
A_{x, b a}=\mathrm{e}^{-\frac{\mu x}{\sigma^{2}}} \sum_{n=1}^{\infty} \frac{a_{n}}{\lambda_{n}} F(b, a, n ; \mu)
$$

where

$$
\begin{aligned}
& F(b, a, n ; \mu)=-K_{1}\left[a \mathrm{e}^{\frac{\mu a}{\sigma^{2}}} \cos (n \pi)-b \mathrm{e}^{\frac{\mu b}{\sigma^{2}}}\right]+2 K_{1}^{2} K_{2}\left[\mathrm{e}_{\sigma^{2}} \cos (n \pi)-\mathrm{e}^{\frac{\mu b}{\sigma^{2}}}\right], \\
& K_{1}=\frac{(a-b)}{n \pi\left(1+K_{2}^{2}\right)} K_{2}=\frac{\mu(a-b)}{\sigma^{2} n \pi} .
\end{aligned}
$$

Proof. See Appendix

Similarly, for the special case of $\mu=0$, we have

Corollary 3.2. Let $b<x<a$. Then for a Brownian motion $\{X(t), t \geqslant 0\}$ with $X(0)=x, \mu=0$, variance coefficient $\sigma^{2}$ and moving in the strip $[b, a]$, the expected area under its trajectory until the escape time is

$$
A_{x, b a}=\frac{4(a-b)^{2}}{\sigma^{2}} \sum_{n=1}^{\infty} \sin \left(\frac{n \pi(x-b)}{a-b}\right) \frac{[b-a \cos (n \pi)]}{(n \pi)^{3}} .
$$

We are now ready to develop the expressions for the operating characteristics of the inventory system. 


\section{Operating characteristics and objective function}

\subsection{Operating characteristics}

Under the proposed policy there is no intervening action on the inventory levels during the lead time period. Hence, the inventory level behaves as an unrestricted Brownian motion process throughout the lead time period with drift $\mu$ and variance coefficient $\sigma^{2}$. When the order is received, however, the policy may dictate an intervention. Specifically, if the inventory level is found to exceed $S$, an immediate disposal action is taken to reduce the inventory level immediately to $s$; otherwise, no action is taken. Therefore, there may be a sudden jump in the inventory level at the end of lead time period in some cycles. Thus, the end of a lead time period corresponds to the instance when the outstanding order arrives and an immediate disposal action is taken, if necessary. The inventory level at the end of the lead time period is denoted by $x$. As discussed, we reasonably assume $r<x<S$.

In the remaining part of the cycle, the inventory level behaves as a Brownian motion (with drift $\mu$ and variance coefficient $\sigma^{2}$ ) moving within strips, defined by the parameters of the control policy. (See Figs. 1-7 for hypothetical realizations of various cycle types.) Due to an abrupt regime change right after the lead time, we develop the expressions for the operating characteristics of the inventory system, separately, for the lead time period and for the segment after the order arrives until the end of the cycle. Throughout, we let the position of the inventory level at time $t$ be denoted by $I(t)$.

\subsection{Analysis of the lead time period}

We begin with the analysis within a lead time period. As we employ a renewal theoretic approach, without loss of generality, we will set the time origin $t=0$ as the beginning of a regenerative cycle, which, by definition, coincides with the beginning of the lead time period. Since, under the $(S, s, r, Q)$ policy, no replenishment-disposal action is taken during the lead time period, the inventory process behaves as an unrestricted Brownian motion within this period. Therefore, for $0 \leqslant t<L, I(t)$ is normally distributed with mean $(r+\mu t)$ and variance $\sigma^{2} t$, where $r$ is the initial position of the process in a cycle (see [8]). We assume that $\mu$ takes only zero or negative values. Hence, the expected on-hand inventory carried during the lead time period, $E(\mathrm{LOH})$, and the expected time that the inventory system is out of stockout in a cycle, $E(\mathrm{SO})$, can be computed as follows:

$$
E(\mathrm{LOH})=\int_{0}^{L} E\left([I(t)]^{+}\right) \mathrm{d} t
$$

with

$$
E\left([I(t)]^{+}\right)=\int_{0}^{\infty} x f_{I(t)}(x) \mathrm{d} x=\sqrt{\frac{\sigma^{2} t}{2 \pi}} \mathrm{e}^{-\frac{(r+\mu t)^{2}}{2 \sigma^{2} t}}+(r+\mu t) \Phi\left(\frac{r+\mu t}{\sqrt{\sigma^{2} t}}\right),
$$

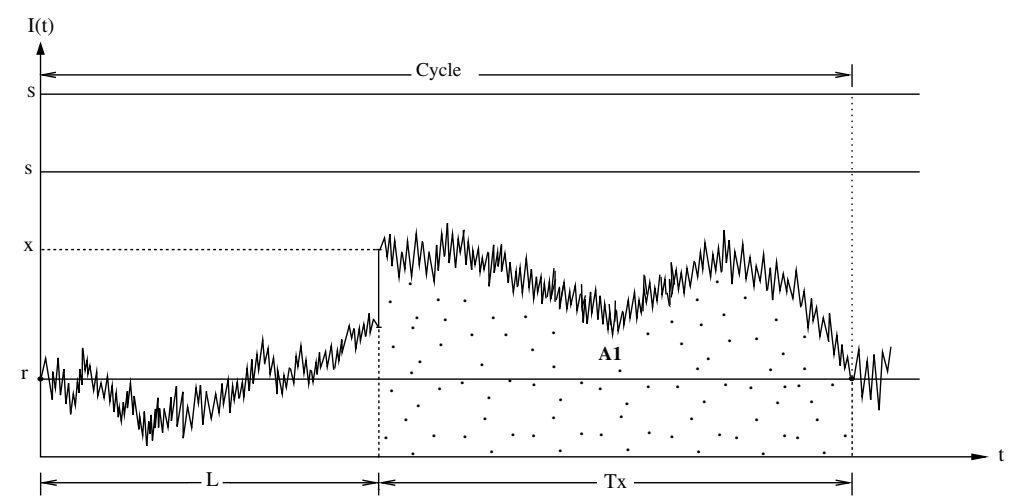

Fig. 1. Possible realization of the inventory level process for $r<x \leqslant s$. 


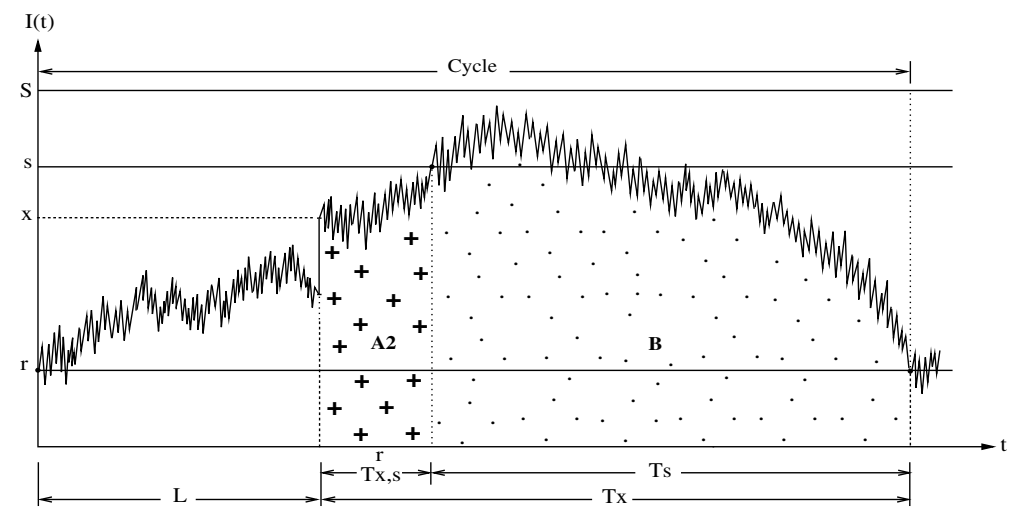

Fig. 2. Possible realization of the inventory level process for $r<x \leqslant s$.

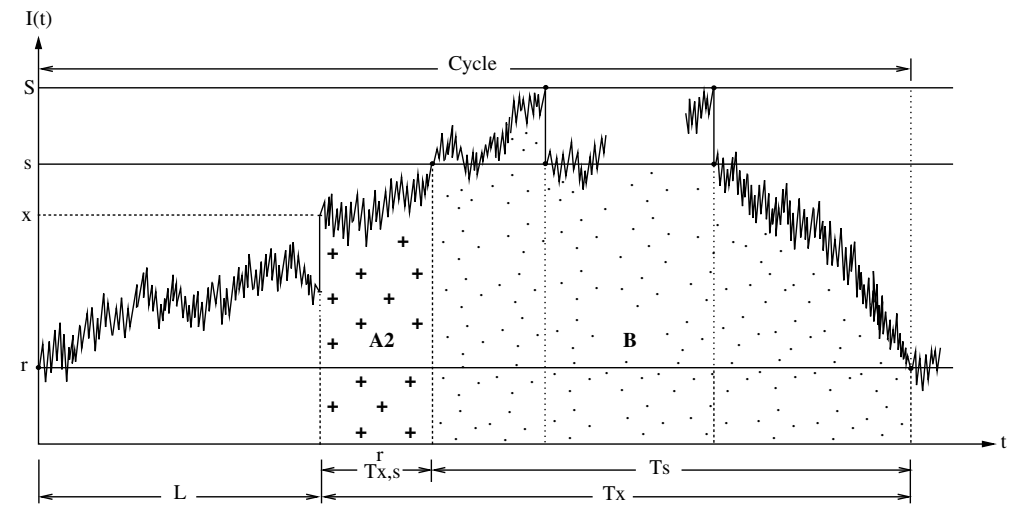

Fig. 3. Possible realization of the inventory level process for $r<x \leqslant s$.

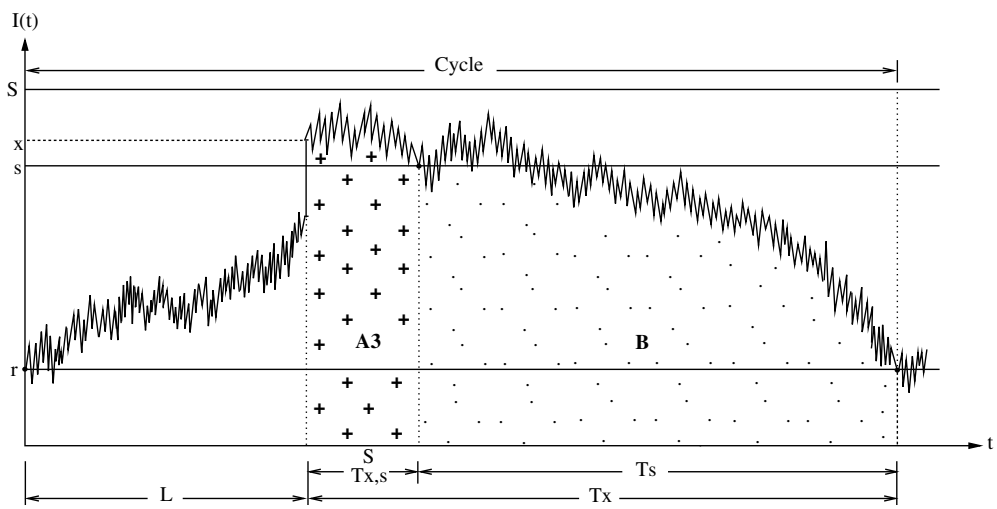

Fig. 4. Possible realization of the inventory level process for $s<x<S$.

where $f_{I(t)}(x)$ denotes the probability density of $I(t)$ and $\Phi$ is the cumulative distribution function of a standard normal distribution. Substituting (6) in (5) yields the following result:

$$
E(\mathrm{LOH})=\int_{0}^{L} \sqrt{\frac{\sigma^{2} t}{2 \pi}} \mathrm{e}^{-\frac{(r+\mu)^{2}}{2 \sigma^{2} t}} \mathrm{~d} t+r \int_{0}^{L} \Phi\left(\frac{r+\mu t}{\sqrt{\sigma^{2} t}}\right) \mathrm{d} t+\mu \int_{0}^{L} t \Phi\left(\frac{r+\mu t}{\sqrt{\sigma^{2} t}}\right) \mathrm{d} t .
$$




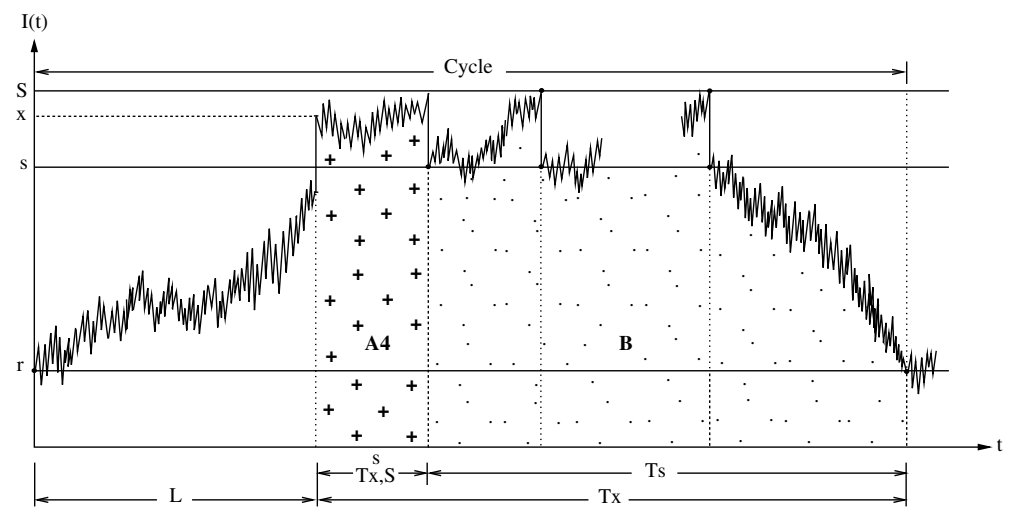

Fig. 5. Possible realization of the inventory level process for $s<x<S$.

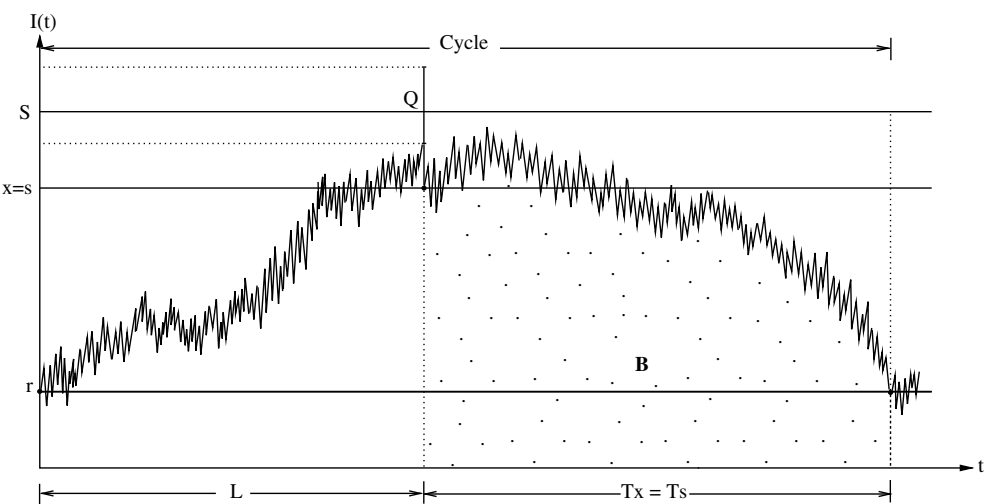

Fig. 6. Possible realization of the inventory level process for $x=s$.

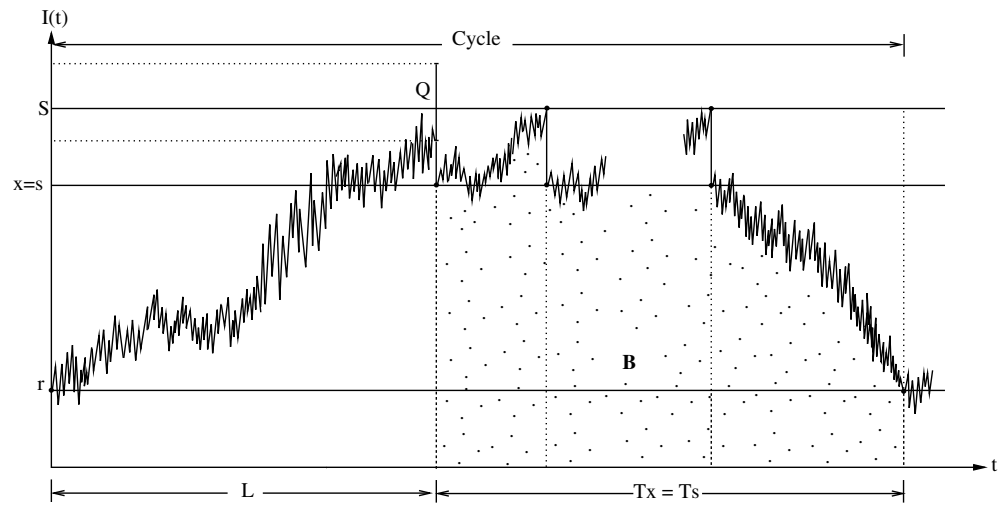

Fig. 7. Possible realization of the inventory level process for $x=s$.

Similarly, the expected stockout period during the lead time is given by

$$
\begin{aligned}
& E(\mathrm{SO})=\int_{0}^{L} P(I(t) \leqslant 0) \mathrm{d} t=\int_{0}^{L}\left[1-\Phi\left(\frac{r+\mu t}{\sqrt{\sigma^{2} t}}\right)\right] \mathrm{d} t \\
& =L-\int_{0}^{L} \Phi\left(\frac{r+\mu t}{\sqrt{\sigma^{2} t}}\right) \mathrm{d} t .
\end{aligned}
$$




\subsection{Analysis after the lead time period}

We first derive the expressions for the operating characteristics conditional on the inventory level $x$ after the lead time period ends and denote them by subscript $x$. Later we will uncondition them to obtain the operating characteristics of the system. In this segment of the cycle, the behavior of $I(t)$ is identical to that of a Brownian motion moving in strips. Therefore, our analysis rests on the results given in Section 3.

Under the proposed control policy, whenever the inventory level exceeds $S$, the excess inventory is disposed of and the inventory level is brought down to $s$. Since the material inflow and demand processes are random, there may be a multiple of such disposal actions before the cycle ends, leading to a possible repetitive behavior for the process within a regenerative cycle (e.g. Figs. 3, 5 and 7).

Fig. 1 illustrates an example of a trajectory where the process, starting at some level $x(r<x \leqslant s)$, escapes from the strip $[r, s]$ for the first time through the lower-boundary $r$ and completes the cycle. However, if the first escape occurs at the upper boundary, $s$, then the inventory process initiates a new movement within the strip $[r, S]$ starting from $s$. Here again, if the process hits $r$ before $S$, then the cycle is completed (see Fig. 2). Otherwise, at the instance of hitting $S$, a disposal occurs to make the process jump down to $s$, restarting a probabilistically identical movement. Such restarts may occur repeatedly; Fig. 3 depicts an example. (Similar examples for the case of an immediate disposal action after the order arrives are illustrated in Figs. 6 and 7.)

These repeated restarts generate a maze-type (i.e. recurrent) behavior which we exploit in deriving the operating characteristics of interest. In particular, we will derive the expressions for the expected cycle length, expected inventory-days (i.e., inventory carried over time) after the lead time period and the expected number of disposal actions and the quantity disposed of in a cycle by considering the behavior of the inventory level as a Brownian motion moving in strips and invoking some of the key results presented in Section 3. Since the underlying process is a Brownian motion, we consider each strip separately and, for convenience, take the time origin within that strip as the time instance when the process enters that strip. We, then, patch such strips together to obtain the behavior within the totality of the cycle.

We begin our analysis with the expected time until the cycle ends. Define $T_{u}$ as the remaining time until the end of a regenerative cycle from any instance when the inventory level is $u$. Then, $T_{x}$ will be the time until the cycle is completed if the inventory level after the lead time period is $x$.

Case 1: $r<x \leqslant s$. According to the possible cycle realizations discussed above, for $x \in(r, s]$ we can write

$$
\begin{aligned}
T_{x} & = \begin{cases}T_{x, r} & \text { if } T_{x, r}<T_{x, s}, \\
T_{x, s}+T_{s} & \text { if } T_{x, s}<T_{x, r},\end{cases} \\
& =T_{x, r s}+T_{s} \chi\left(T_{x, s}<T_{x, r}\right),
\end{aligned}
$$

where $\chi(\cdot)$ is the indicator function and

$$
\begin{aligned}
T_{s} & = \begin{cases}T_{s, r} & \text { if } T_{s, r}<T_{s, S}, \\
T_{s, S}+T_{s} & \text { if } T_{s, S}<T_{s, r},\end{cases} \\
& =T_{s, r S}+T_{s} \chi\left(T_{s, S}<T_{s, r}\right)
\end{aligned}
$$

In (10), the first line corresponds to a realization where the process leaves the strip $[r, s]$ at level $r$, completing the cycle; and, the second line corresponds to a realization where the process leaves the strip at $s$, starting a motion in a new strip $[r, S]$, for which the time until the cycle ends is given by (12). Noting the maze-type behavior discussed above, we find

$$
E\left(T_{x}\right)=E\left(T_{x, r s}\right)+E\left(T_{s}\right) P_{x, s}^{r},
$$

where

$$
E\left(T_{s}\right)=\frac{E\left(T_{s, r S}\right)}{P_{s, r}^{S}} .
$$

Using Proposition 3.2 and the above relations, the expected time remaining until the end of a cycle with $x$ units of on-hand inventory after the lead time period is given as follows: 


$$
E\left(T_{x}\right)=-\frac{(x-r)}{\mu}+\frac{(S-s)}{\mu} \cdot \frac{\left(\mathrm{e}^{-\frac{2 \mu x}{\sigma^{2}}}-\mathrm{e}^{-\frac{2 \mu r}{\sigma^{2}}}\right)}{\left(\mathrm{e}^{-\frac{2 \mu S}{\sigma^{2}}}-\mathrm{e}^{-\frac{2 \mu s}{\sigma^{2}}}\right)} .
$$

Case 2: $s<x<S$. The remaining cycle time at the end of the lead time for this case is written as

$$
T_{x}=T_{x, s S}+T_{s} \text {. }
$$

The first term in the above expression is the time until the process escapes from the strip $[s, S]$; the second term comes from the fact that regardless of the boundary from which the process escapes, the remaining cycle time is given by $T_{s}$. Again invoking Proposition 3.2 to obtain the expected value of the expression given in (15), we find that this expectation is exactly the same as the one given by (14).

Next, we consider the expected inventory-days (the area under the inventory level curve) within a cycle after the lead time period.

Let $\mathrm{OH}_{u}$ be the inventory carried until the end of the cycle from any instance when the inventory level is $u$. Also, let $\mathrm{OH}_{u, v}^{z}$ be the inventory carried until the inventory level hits $v$ before $z$ for the first time starting at $u$ and $\mathrm{OH}_{u, v z}$ be the inventory carried until the process escapes from strip $[v, z]$. We again consider the cases separately:

Case 1: $r<x \leqslant s$. The inventory carried until the end of the cycle if the inventory level after the lead time is $x$, can be written as follows:

$$
\mathrm{OH}_{x}= \begin{cases}\mathrm{OH}_{x, r}^{s} & \text { if } T_{x, r}<T_{x, s}, \\ \mathrm{OH}_{x, s}^{r}+\mathrm{OH}_{s} & \text { if } T_{x, s}<T_{x, r}\end{cases}
$$

The first line of (16) corresponds to the area denoted by $A 1$ in Fig. 1, where the process hits $r$ before $s$. The second line corresponds to the case where $s$ is hit before $r$ (see Figs. 2 and 3). The first term of the second line is the area, $A 2$, whereas the second one is, $B$, the total inventory carried until the end of the cycle from the point $s$, denoted by $\mathrm{OH}_{s}$.

Similar to the cycle length arguments, we see that there exists a maze-type structure in the carried inventory expressions. Hence, we have

$$
E\left(\mathrm{OH}_{x}\right)=E\left(\mathrm{OH}_{x, r s}\right)+E\left(\mathrm{OH}_{s}\right) P_{x, s}^{r},
$$

where

$$
E\left(\mathrm{OH}_{s}\right)=\frac{E\left(\mathrm{OH}_{s, r S}\right)}{P_{s, r}^{S}} .
$$

Case 2: $s<x<S$. In this case, we have

$$
\mathrm{OH}_{x}= \begin{cases}\mathrm{OH}_{x, s}^{S}+\mathrm{OH}_{s} & \text { if } T_{x, s}<T_{x, S}, \\ \mathrm{OH}_{x, S}^{s}+\mathrm{OH}_{s} & \text { if } T_{x, S}<T_{x, s}\end{cases}
$$

Similarly, the expressions $\mathrm{OH}_{x, s}^{S}$ and $\mathrm{OH}_{x, S}^{s}$ given by (17) correspond to the areas $A 3$ and $A 4$ in Figs. 4 and 5 , respectively. Therefore, we can write as before

$$
E\left(\mathrm{OH}_{x}\right)=E\left(\mathrm{OH}_{x, s S}\right)+E\left(\mathrm{OH}_{s}\right) \text {. }
$$

In order to evaluate the above expectations, we employ the result given by Proposition 3.3 in which the term $A_{x, b a}$ corresponds to the expected inventory carried until the process escapes from strip $[b, a]$ starting from $x$. Thus, we have $E\left(\mathrm{OH}_{x, r S}\right)=A_{x, r S}$ and $E\left(\mathrm{OH}_{s, r S}\right)=A_{s, r S}$.

Next, we develop the expressions for the expected number of disposal actions, each incurring a fixed cost of $K_{\mathrm{d}}$, and the expected quantity disposed of with each disposal action. Under the proposed policy, whenever the inventory level exceeds the disposal threshold $S$, excess amount is disposed of, as long as there are no outstanding orders. Therefore, the expected number of disposal actions and the total number of units disposed of are evaluated only for the remainder of the cycle after the order arrives. Since the inventory levels may grow excessively during a lead time period due to inflow of material, it is possible that, just when the order arrives, the inventory level, $I(L)+Q$, may be found at or above $S$. If so, a disposal action is taken immediately reduc- 
ing the inventory level instantaneously to $s$ and the quantity disposed of is $(I(L)+Q-s)$. Such an immediate disposal occurs with probability $P(I(L)+Q \geqslant S)$, where $I(L)$ is normally distributed with mean $\mu L+r$ and variance $\sigma^{2} L$. In this case, the remaining segment of the cycle begins with inventory level $x=s$ with the positive probability given above. (See Figs. 6 and 7.) If, however, $I(L)+Q$ is found to be less than $S$, then, no disposal action is taken and the remainder of the cycle begins with the inventory level $x=I(L)+Q$ with the probability density of a normal variate with mean $\mu L+r+Q$ and variance $\sigma^{2} L$. Due to the one order outstanding assumption, we assume the probability that $x \leqslant r$ is negligibly small, and, hence, consider only the case when $r<x<S$. Let $N_{x}$ be the number of disposal actions until the end of the cycle, if the inventory level after the lead time is $x$. Observe that $x$ may attain the value $s$ in two ways: Either when an immediate disposal action is taken after the order arrives, or when the level of the unrestricted Brownian motion immediately before the order arrives is found to be $s-Q$. The first case has the positive probability given above, whereas the latter is described by a normal probability density. We consider these cases separately.

First, consider the realizations when there is no immediate disposal action after an order arrives. Starting at $x$, if the process directly goes down to $r$, without hitting $S$, then $N_{x}=0$ with probability $P_{x, r}^{S}$. In order that there are $n$ disposals, the process, starting from $x$, first goes to $S$ without hitting $r$ with probability $P_{x, S}^{r}$ and the inventory level is immediately reduced to level $s$ by disposal. Then, the process, starting at $s$ reaches $S$ before $r$ with probability $P_{s, S}^{r}$ consecutively for $n-1$ times, and finally, starting at $s$, it reaches the level $r$ before $S$ and completes the cycle. Hence, we can write the following probability mass function for $N_{x}$ :

$$
P\left(N_{x}=n\right)=\left\{\begin{array}{llc}
P_{x, r}^{S} & \text { if } & n=0, \\
P_{x, S}^{r} P_{s, r}^{S}\left(P_{s, S}^{r}\right)^{(n-1)} . & \text { if } & n>0
\end{array}\right.
$$

Then, the expected number of disposal actions when the inventory level at the end of the lead time is $x$, is given by the following expression for which the proof is given in Appendix.

$$
E\left(N_{x}\right)=\frac{\mathrm{e}^{-\frac{2 \mu x}{\sigma^{2}}}-\mathrm{e}^{-\frac{2 \mu r}{\sigma^{2}}}}{\mathrm{e}^{-\frac{2 \mu S}{\sigma^{2}}}-\mathrm{e}^{-\frac{2 \mu s}{\sigma^{2}}} .}
$$

In each disposal action, the quantity disposed of is exactly $(S-s)$; therefore, the expected total amount of stock disposed of in a cycle with $x$ units of on hand stock at the end of the lead time period can be found by multiplying this number by $E\left(N_{x}\right)$.

Next, consider the realizations where an immediate disposal is made after an order arrives; that is, $x=s$ with a positive probability. In this case, the number of disposal actions is one more than the case when $x=s$ without any disposal action. In the particular disposal action immediately after the order arrives, the number of units disposed of depends on the inventory level immediately before the order arrives. Let $n(t ; u, v)$ and $\bar{N}(t ; u, v)=\int_{t}^{\infty} n(x ; u, v) \mathrm{d} x$ denote henceforth the probability density and the tail probability of a normal random variable with mean $u$ and variance $v$. Then, since $I(L)$ is normally distributed with mean $r+\mu L$ and variance $\sigma^{2} L$, the expected number of units disposed of immediately after the order arrives is given by

$$
\frac{\int_{(S-Q)}^{\infty}(y+Q-s) n\left(y ; r+\mu L, \sigma^{2} L\right) \mathrm{d} y}{\bar{N}\left(S-Q ; r+\mu L, \sigma^{2} L\right)} .
$$

\subsection{Special case of zero drift $(\mu=0)$}

For the case of perfect balance between the external demand and autonomous supply rates, some expressions of the operating characteristics yield simple elegant expressions provided below:

$$
\begin{aligned}
& E(\mathrm{LOH})=\int_{0}^{L} \sqrt{\frac{\sigma^{2} t}{2 \pi}} \mathrm{e}^{-\frac{r^{2}}{2 \sigma^{2}}} \mathrm{~d} t+r \int_{0}^{L} \Phi\left(\frac{r}{\sqrt{\sigma^{2} t}}\right) \mathrm{d} t, \\
& E(\mathrm{SO})=L-\int_{0}^{L} \Phi\left(\frac{r}{\sqrt{\sigma^{2} t}}\right) \mathrm{d} t
\end{aligned}
$$




$$
\begin{aligned}
& E\left(T_{x}\right)=\frac{(x-r)(S+s-x-r)}{\sigma^{2}}, \\
& E\left(\mathrm{CL}_{x}\right)=L+\frac{(x-r)(S+s-x-r)}{\sigma^{2}},
\end{aligned}
$$

where $E\left(\mathrm{CL}_{x}\right)$ denotes the expected cycle length conditioned on $x$. Finally, for $r<x<S$, the expected number of disposals given by (19) reduces to

$$
E\left(N_{x}\right)=\frac{x-r}{S-s} .
$$

The expressions for $E\left(\mathrm{OH}_{x}\right)$ for Cases 1 and 2 are obtained by employing the result given by Corollary 3.2. Similarly, for $x=s$, the expressions for the expected number of disposal actions and the quantities disposed of are found as discussed above by setting $\mu=0$.

\subsection{Objective function}

The expected total cost under the $(S, s, r, Q)$ policy can now be obtained by using the equations above. The operating characteristics we have derived so far were conditional on the level of the inventory at the end of the lead time period. Therefore, we first express the unconditional expectations, and then, construct the expected cost rate. The distribution of $x$ has both a continuous component described by a normal density and a discrete point, the probability of which is given by the tail probability of a normal random variable.

The expected cycle length, $E(\mathrm{CL})$, and the expected on-hand inventory carried during a cycle, $E(\mathrm{OH})$, are given by

$$
\begin{aligned}
& E(\mathrm{CL})=L+\int_{r}^{S} E\left(T_{x}\right) n\left(x ; r+\mu L+Q, \sigma^{2} L\right) \mathrm{d} x+E\left(T_{s}\right) \bar{N}\left(S ; r+\mu L+Q, \sigma^{2} L\right) \\
& E(\mathrm{OH})=E(\mathrm{LOH})+\int_{r}^{S} E\left(\mathrm{OH}_{x}\right) n\left(x ; r+\mu L+Q, \sigma^{2} L\right) \mathrm{d} x+E\left(\mathrm{OH}_{s}\right) \bar{N}\left(S ; r+\mu L+Q, \sigma^{2} L\right)
\end{aligned}
$$

The expected disposal cost, $E(\mathrm{DC})$, which includes both fixed and variable components, is similarly found as

$$
\begin{aligned}
E(\mathrm{DC})= & {\left[K_{\mathrm{d}}+c_{\mathrm{d}}(S-s)\right] \int_{r}^{S} E\left(N_{x}\right) n\left(x ; r+\mu L+Q, \sigma^{2} L\right) d x+\left[K_{\mathrm{d}}+c_{\mathrm{d}}(S-s)\right] E\left(N_{s}\right) \bar{N}\left(S ; r+\mu L+Q, \sigma^{2} L\right) } \\
& +K_{\mathrm{d}} \bar{N}\left(S ; r+\mu L+Q, \sigma^{2} L\right)+c_{\mathrm{d}} \int_{(S-Q)}^{\infty}[y+Q-s] n\left(y ; r+\mu L, \sigma^{2} L\right) \mathrm{d} y
\end{aligned}
$$

In a cycle, the total inflow of returns and replenished units must equal the total outflow of external demand and disposed of units. Hence,

$$
\begin{aligned}
E(\mathrm{DC})= & K_{\mathrm{d}} \int_{r}^{S} E\left(N_{x}\right) n\left(x ; r+\mu L+Q, \sigma^{2} L\right) \mathrm{d} x+K_{\mathrm{d}} E\left(N_{s}\right) \bar{N}\left(S ; r+\mu L+Q, \sigma^{2} L\right)+K_{\mathrm{d}} \bar{N}\left(S ; r+\mu L+Q, \sigma^{2} L\right) \\
& +c_{\mathrm{d}}\left[\left(\mu_{R}-\mu_{D}\right) E(\mathrm{CL})+Q\right]
\end{aligned}
$$

where the last term is unit disposal cost multiplied by the expected quantity disposed of in a cycle, $E(D Q)$.

Invoking the Renewal Reward Theorem (see [18]), the expected total cost rate is given by the ratio of the expected cycle cost to the expected cycle length yielding

$$
\mathrm{TC}(S, s, r, Q)=\frac{c_{\mathrm{o}} Q+K_{\mathrm{o}}+h E(\mathrm{OH})+E(\mathrm{DC})}{E(\mathrm{CL})}+c_{r} \mu_{R}
$$

After some algebra, we get

$$
\begin{aligned}
\operatorname{TC}(S, s, r, Q)= & \frac{\left[c_{\mathrm{o}}-c_{r}\right] Q+K_{\mathrm{o}}+h E(O H)+K_{\mathrm{d}}\left\{\int_{r}^{S} E\left(N_{x}\right) n\left(x ; r+\mu L+Q, \sigma^{2} L\right) \mathrm{d} x\right.}{E(C L)} \\
& +\frac{\left.\left[E\left(N_{s}\right)+1\right] \bar{N}\left(S ; r+\mu L+Q, \sigma^{2} L\right)\right\}}{E(C L)}+\frac{\left[c_{\mathrm{d}}+c_{r}\right] E(D Q)}{E(C L)}+c_{r} \mu_{D}
\end{aligned}
$$


The optimization problem is then stated as

$$
\begin{array}{ll}
\min & \mathrm{TC}(S, s, r, Q), \\
\text { s.t. } & \frac{E(\mathrm{SO})}{E(\mathrm{CL})} \leqslant 1-\alpha .
\end{array}
$$

In the foregoing model, we make a couple of basic assumptions: (i) there is at most one outstanding order at any time and, (ii) the on-hand inventory at the end of a lead time period is always greater than the reorder point, $x>r$. Next, we briefly discuss their impact on our analysis. Under (i), we underestimate the expected on-hand inventory, the expected ordering/purchasing costs and the fill rate. As the inventory level at the end of the lead time period is also being overestimated, it is likely that we are underestimating the expected number of disposals and ensuing disposal costs. For high desired fill rates, the assumption of at most one outstanding order would hold well since $r$ would be set accordingly high. Assumption (ii) holds when the probability that the net demand during lead time exceeds the order quantity $Q$ is very small. If this assumption does not hold, it would imply in general that the expected on-hand inventory during the lead time and the fill rate are overestimated, resulting in underestimation of the ordering/purchasing costs and overestimation of disposal costs.

In our numerical study, we also adopted an approximation to alleviate the numerical tedium, by replacing the inventory level at the end of the lead time by its expected value. This approximation is similar to the one commonly used for the classical $(Q, r)$ model where it is assumed that, on average, only the 'safety stock' component of the reorder point level remains at the end of lead time (see [14]). Hence, we set $x=Q+r+\mu L$ in the expected cycle cost and cycle length expressions given in Section 4. In a preliminary numerical investigation on 270 instances, we compared the approximate and the exact expected cost rates. The return ratio and coefficients of variation of return and demand processes are denoted by $\gamma\left(=\mu_{\mathrm{R}} / \mu_{\mathrm{D}}\right), \mathrm{CV}_{\mathrm{R}}\left(=\sigma_{\mathrm{R}} / \mu_{\mathrm{R}}\right)$ and $\mathrm{CV}_{\mathrm{D}}\left(=\sigma_{\mathrm{D}} / \mu_{\mathrm{D}}\right)$, respectively. The instances were generated from a set where $h=1, c_{r}=4, c_{\mathrm{d}}=1, \mu=-20$, $L=0.1, \mathrm{CV}_{\mathrm{D}}=0.3, \alpha \in\{0.95,0.99,0.999\}, K_{\mathrm{d}} / K_{\mathrm{o}} \in\{0.1,0.2,0.5\}, K_{\mathrm{o}} \in\{500,1000\}, \mathrm{CV}_{\mathrm{R}} \in\{0.5,1,2\}$ and $\gamma$ varying between 0.50 and 0.95 at increments of 0.05 . Overall, the mean and the median of the percentage deviation between the exact and approximate computations were -0.27 and $-0.19 \%$, respectively. Thus, we conclude that this approximation would not confound the main implications of our study.

\section{Numerical study}

We conducted our numerical study to investigate two aspects: (i) the sensitivity of the control policy parameters to various system parameters, and (ii) the comparison of the proposed policy with the disposal option vis a vis the classical $(Q, r)$ policy without such an option.

The test bed used in our numerical study was generated with the following cost parameters: $h=1$, $K_{\mathrm{o}} \in\{50,100,500,1000\}, K_{\mathrm{d}} / K_{\mathrm{o}} \in\{0.05,0.1,0.2,0.25,0.5\}$ and unit disposal cost, $c_{\mathrm{d}}$, is taken as $11-400 \%$ of the value of a new item, $c_{\mathrm{o}}\left(c_{\mathrm{d}}=1,2,4 ; c_{\mathrm{o}}+c_{\mathrm{d}}=5,10\right)$. We set $c_{r}=c_{\mathrm{o}}$ so that arbitrage opportunities are not allowed. We considered $L \in\{0.1,1,5\}, \alpha \in\{0.95,0.99,0.999\}, \gamma$ over a range of 0.50 to 0.97 and $\mu \in\{0,-1,-20,-200,-2000\}$. (Taking into account $\gamma$ and annual cost of capital of, say, around $10 \%$, the actual demand rates considered would range between 2 and 40,000 per year.) For large net demand rates $(\mu \in\{-20,-200,-2000\})$, we also tested $K_{\mathrm{o}} /|\mu| \in\{2.5,5,25,50\}$ which result in alike inventory turnover rates, while retaining the rest of the parameter set. For a given non-zero net demand rate, $\sigma$ can be calculated from the relationship between $\gamma, \mathrm{CV}_{\mathrm{R}}$ and $\mathrm{CV}_{\mathrm{D}}$; that is, $\sigma=\frac{|\mu|}{1-\gamma} \sqrt{\left[\gamma \mathrm{CV}_{\mathrm{R}}\right]^{2}+\mathrm{CV}_{\mathrm{D}}^{2}}$. Thus, for non-zero net demand rates, a broad set of $\sigma$ values are obtained by setting $\mathrm{CV}_{\mathrm{R}} \in\{0.5,0.75,1,1.5,2\}$ and $\mathrm{CV}_{\mathrm{D}}=0.3$ for different $\gamma$. Although it does not confound our results in any way, we chose our parameters such that the return process is more variable than the external demand process. This would be the case when the return process is determined by the consumers' environmental awareness and propensity to return reusables. Since these propensities would not be uniform across the consumer population, they would add to the uncertainties of the return process.

For the special case of no drift, $\mu=0$, we only examined the cases where $\mathrm{CV}_{\mathrm{D}}=0.3, \mathrm{CV}_{\mathrm{R}}=0.4$ and $\sigma=1$. With this construction, we generated a total of 24,912 instances: (i) 324 instances for $\mu=0$, (ii) 24,300 
instances for $\mu=-1$ and -20 and (iii) 288 additional instances for $\mu=-200$ and -2000 . (The entire data set and numerical results can be obtained from the authors.)

Our experimental set consists of realistic scenarios as well as somewhat extreme situations for sensitivity analysis. For example, with $\mu=-200, \gamma=0.95, L=0.1, c_{\mathrm{d}}=1$ and $c_{r}=9$ we have a scenario where, for an annual cost of capital of slightly more than $11 \%$, the demand rate is approximately 4000 units per year, the delivery lead time is about 5 weeks and unit disposal cost is almost $10 \%$ of a new product - a realistic high demand, low unit disposal cost setting. However, with $\mu=0, \mathrm{CV}_{\mathrm{D}}=0.3, \mathrm{CV}_{\mathrm{R}}=0.4, \sigma=1, L=0.1, c_{\mathrm{d}}=1$ and $c_{r}=4$, we would be considering a scenario where, for an annual cost of capital of $25 \%$, the demand rate is only 2 units per year and unit disposal cost is a quarter of the cost of a new item - an extreme setting with slow-moving items and high holding costs. Hence, we are able to observe the impact of disposals with fixed costs over a large range of demand settings. Throughout, we use $(*)$ to denote the optimal value of a policy parameter or of an entity of interest.

\subsection{Sensitivity analysis}

We begin our discussion with the special case of $\mu=0$, which corresponds to a return ratio of one. A perfectly-balanced system provides an interesting benchmark case and highlights the fundamental tradeoff between inventory concerns and the disposal option. In Table 1, we provide results as we vary the maximum inventory level $S$ at different values. Setting $S$ externally enables us to observe the impact of disposal thresholds and may as well be viewed as corresponding to a physical constraint on the inventory system such as limited warehouse or storage capacities. First, we observe that the expected total cost rate is unimodal in $S$. (See also Fig. 8.) Incidentally, our numerical studies indicate joint unimodality of the expected cost rate function with respect to the policy parameters for all net demand rates, although we have not been able to show this analytically. A second observation from Table 1 concerns the appropriateness of the $(Q, r)$ policy. By definition, as $S \rightarrow \infty$, the proposed policy reduces to the $(Q, r)$ control policy which prohibits any disposal. We see that employing the best $(Q, r)$ policy instead of the best $(S, s, r, Q)$ policy more than triples the cost rates for the no drift case in this setting! We shall discuss policy comparison in detail further below. We proceed to discuss the results on sensitivity of the control policy parameter for demand settings with negative drift.

From the results presented in Table 2, we observe that the overall behavior of the policy parameters with respect to the system parameters is intuitive. As the fixed disposal cost, $K_{\mathrm{d}}$, increases, $S^{*}$ increases, whereas, $s^{*}$ decreases. This behavior can be explained as follows. As $K_{\mathrm{d}}$ increases, a disposal decision becomes more costly. The system should either reduce the number of instances of disposal or increase the quantity disposed of in each instance. Hence, we observe that $\left(S^{*}-s^{*}\right)$ increases. Similarly, as $K_{\mathrm{d}}$ increases, $Q^{*}$ decreases to prevent possible disposals, which become more expensive.

The net demand variability is governed by $\gamma$ and $C V_{R}$ in our numerical experiments. The impact of each on the policy parameters is similar. As $\gamma$ increases, both $Q^{*}$ and $\left(S^{*}-s^{*}\right)$ tend to increase. This implies that larger net demand variability results in more disposals. As expected, the reorder point is also increasing in $\gamma$ since $r^{*}$ implicitly depends on the fill rate.

The impact of the desired fill rate is not monotonic on $S^{*}, s^{*}$ and $Q^{*}$; it appears that other system parameters as $K_{\mathrm{d}}, \gamma$ and $\mu$ also have an effect on them simultaneously. However, the reorder point is monotonically increasing in $\alpha$. This is expected since a larger $r^{*}$ value means a higher fill rate. Sensitivity results for lead time and fixed ordering cost are not tabulated herein for brevity. In these unreported results, similar effects are observed for the lead time.

As to the fixed ordering cost, as $K_{\mathrm{o}}$ increases, the optimal order quantity is observed to increase, as expected. In our model, a larger order quantity implies that the inventory level as a stochastic process is more likely to visit the states in the neighborhood of the disposal trigger level. Hence, with a larger order quantity, the likelihood of a disposal is larger. In order to compensate for this, $S^{*}$ increases, thereby, making less likely the event of hitting the disposal trigger; and $s^{*}$ increases to reduce the incurred variable disposal costs, but not as much as $S^{*}$, so that $\left(S^{*}-s^{*}\right)$ also increases with $K_{\mathrm{o}}$. Hence, one can conclude that the increase in the optimal order quantity is also partly due to an increase in the quantity disposed of. 
Table 1

Sensitivity analysis when $S$ is set exogenously $\left(L=5, \mu=0, \sigma=1, \alpha=0.99, K_{\mathrm{o}}=500, c_{\mathrm{d}}=1, c_{r}=4\right)$

\begin{tabular}{|c|c|c|c|c|c|}
\hline$K_{\mathrm{d}}$ & $S$ & $s^{*}$ & $r^{*}$ & $Q^{*}$ & $\mathrm{TC}^{*}$ \\
\hline 50 & 10 & 7.89 & 2.02 & 6.66 & 27.31 \\
\hline 100 & 10 & 7.90 & 2.04 & 6.13 & 30.00 \\
\hline 250 & 10 & 7.91 & 2.05 & 5.35 & 37.37 \\
\hline 50 & 15 & 10.54 & 0.75 & 9.21 & 21.02 \\
\hline 100 & 15 & 9.88 & 0.86 & 8.59 & 21.70 \\
\hline 250 & 15 & 8.86 & 1.03 & 7.55 & 23.46 \\
\hline 50 & 17 & 10.74 & 0.53 & 9.53 & 20.79 \\
\hline 100 & 17 & 10.21 & 0.61 & 9.06 & 21.22 \\
\hline 250 & 17 & 9.28 & 0.76 & 8.17 & 22.41 \\
\hline 50 & 20 & 10.36 & 0.33 & 9.41 & 20.93 \\
\hline 100 & 20 & 10.08 & 0.37 & 9.16 & 21.18 \\
\hline 250 & 20 & 9.48 & 0.47 & 8.59 & 21.89 \\
\hline 50 & 25 & 9.38 & 0.13 & 8.80 & 21.68 \\
\hline 100 & 25 & 9.29 & 0.14 & 8.71 & 21.81 \\
\hline 250 & 25 & 9.04 & 0.19 & 8.47 & 22.17 \\
\hline 50 & 40 & 6.31 & 0.00 & 6.09 & 25.24 \\
\hline 100 & 40 & 6.30 & 0.00 & 6.09 & 25.27 \\
\hline 250 & 40 & 6.31 & 0.00 & 6.09 & 25.38 \\
\hline 50 & 200 & 1.61 & 0.00 & 1.57 & 75.74 \\
\hline 100 & 200 & 1.61 & 0.00 & 1.57 & 75.74 \\
\hline 250 & 200 & 1.61 & 0.00 & 1.57 & 75.74 \\
\hline
\end{tabular}

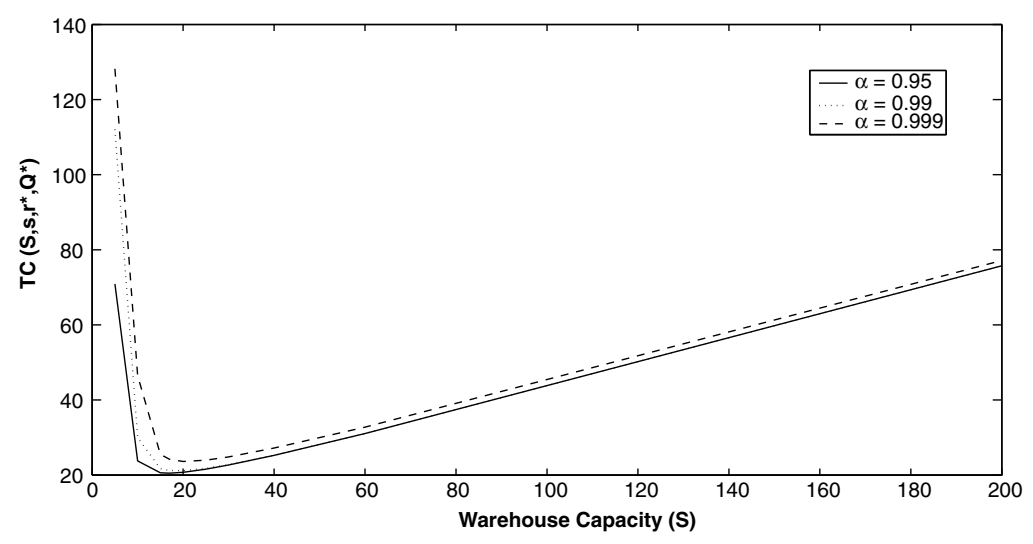

Fig. 8. Total cost rate vs. warehouse capacity $(\mu=0, \sigma=1)$.

\subsection{Comparison with the $(Q, r)$ Policy}

Next, we compare the performance of the system under the proposed control policy with the disposal option vis a vis that under the classical $(Q, r)$ policy. Our goal in this part of the numerical study is to investigate the utility of the disposal option in inventory control of reusables and to assess the impact on costs of ignoring such an option. We do not report the values of the parameters of the best $(Q, r)$ policy but only present the percentage cost difference between the two policies, $\Delta \%=\frac{\mathrm{TC}_{\left(Q^{*}, r^{*}\right)}-\mathrm{TC}_{\left(S^{*}, s^{*}, Q^{*}\right)}}{\mathrm{TC}_{\left(Q^{*}, r^{*}\right)}} \times 100$. A positive value for $\Delta \%$ indicates the savings obtained by employing the proposed policy which allows for disposals. Some of the cost comparisons have been tabulated in Tables 2-5.

As the fixed disposal cost $K_{\mathrm{d}}$ increases, the difference between the two policies decreases. This is best observed in Table 2. This is to be expected since the disposal option becomes more costly. Recall that the 
Table 2

Sensitivity analysis $\left(L=0.1, \mu=-20, K_{\mathrm{o}}=1000\right.$ and $\left.\mathrm{CV}_{\mathrm{R}}=2\right)$

\begin{tabular}{|c|c|c|c|c|c|c|c|c|c|c|}
\hline \multirow[b]{2}{*}{$\alpha$} & \multirow[b]{2}{*}{$\gamma$} & \multirow[b]{2}{*}{$K_{\mathrm{d}}$} & \multicolumn{6}{|c|}{$c_{\mathrm{d}}=1, c_{r}=9$} & \multicolumn{2}{|c|}{$c_{\mathrm{d}}=4, c_{r}=6$} \\
\hline & & & $S^{*}$ & $s^{*}$ & $r^{*}$ & $Q^{*}$ & $\mathrm{TC}^{*}$ & $\Delta \%$ & $\mathrm{TC}^{*}$ & $\Delta \%$ \\
\hline \multirow{15}{*}{0.95} & \multirow{4}{*}{0.95} & 100 & 3302.65 & 2594.43 & 0.00 & 423.21 & 6345.72 & 54.32 & 5145.72 & 59.46 \\
\hline & & 200 & 3408.22 & 2515.92 & 0.00 & 421.77 & 6357.59 & 54.24 & 5157.59 & 59.36 \\
\hline & & 500 & 3598.14 & 2387.08 & 0.00 & 418.57 & 6384.33 & 54.04 & 5184.33 & 59.15 \\
\hline & & 100 & 1782.50 & 1351.75 & 0.00 & 324.16 & 3162.48 & 40.24 & 2562.48 & 45.38 \\
\hline & \multirow[t]{2}{*}{0.90} & 200 & 1848.84 & 1306.06 & 0.00 & 322.82 & 3170.08 & 40.09 & 2570.08 & 45.22 \\
\hline & & 500 & 1969.03 & 1232.26 & 0.00 & 319.86 & 3187.08 & 39.77 & 2587.08 & 44.86 \\
\hline & \multirow{4}{*}{0.80} & 100 & 973.67 & 722.04 & 0.00 & 242.82 & 1539.90 & 12.20 & 1239.90 & 14.72 \\
\hline & & 200 & 1014.53 & 697.32 & 0.00 & 241.68 & 1543.32 & 12.01 & 1243.32 & 14.48 \\
\hline & & 500 & 1089.48 & 658.49 & 0.00 & 239.25 & 1550.78 & 11.58 & 1250.78 & 13.97 \\
\hline & & 100 & 698.97 & 522.23 & 0.00 & 211.73 & 999.29 & 2.37 & 799.29 & 2.95 \\
\hline & \multirow[t]{3}{*}{0.70} & 200 & 729.95 & 506.87 & 0.00 & 210.97 & 1000.47 & 2.26 & 800.47 & 2.81 \\
\hline & & 500 & 787.87 & 483.71 & 0.00 & 209.41 & 1002.91 & 2.02 & 802.91 & 2.51 \\
\hline & & 100 & 519.60 & 389.34 & 0.00 & 199.83 & 661.40 & 0.03 & 528.07 & 0.04 \\
\hline & \multirow[t]{3}{*}{0.55} & 200 & 565.72 & 389.00 & 0.00 & 199.79 & 661.43 & 0.03 & 528.10 & 0.03 \\
\hline & & 500 & 614.98 & 390.92 & 0.00 & 199.62 & 661.50 & 0.02 & 528.17 & 0.02 \\
\hline & & 100 & 3782.84 & 2915.76 & 298.15 & 520.31 & 6653.13 & 53.22 & 5453.13 & 58.12 \\
\hline & \multirow[t]{3}{*}{0.95} & 200 & 3783.12 & 2915.74 & 298.14 & 520.29 & 6663.99 & 53.14 & 5463.99 & 58.04 \\
\hline & & 500 & 3965.20 & 2862.43 & 297.61 & 511.34 & 6690.88 & 52.95 & 5490.88 & 57.83 \\
\hline & & 100 & 1960.68 & 1529.90 & 122.73 & 379.60 & 3289.18 & 39.03 & 2689.18 & 43.92 \\
\hline & \multirow[t]{3}{*}{0.90} & 200 & 2026.88 & 1484.10 & 122.56 & 378.30 & 3296.62 & 38.89 & 2696.62 & 43.76 \\
\hline & & 500 & 2146.78 & 1410.02 & 122.17 & 375.44 & 3313.28 & 38.58 & 2713.28 & 43.41 \\
\hline & & 100 & 1051.56 & 771.66 & 50.00 & 251.01 & 1590.02 & 11.69 & 1290.02 & 14.02 \\
\hline \multirow[t]{8}{*}{0.999} & \multirow[t]{3}{*}{0.80} & 200 & 1074.84 & 763.40 & 49.94 & 249.92 & 1593.30 & 11.51 & 1293.30 & 13.81 \\
\hline & & 500 & 1138.44 & 754.75 & 49.64 & 247.49 & 1600.74 & 11.09 & 1300.74 & 13.31 \\
\hline & & 100 & 746.73 & 554.95 & 27.29 & 226.48 & 1027.01 & 2.25 & 827.01 & 2.77 \\
\hline & \multirow[t]{3}{*}{0.70} & 200 & 787.82 & 539.01 & 27.19 & 226.11 & 1028.19 & 2.13 & 828.19 & 2.64 \\
\hline & & 500 & 822.16 & 530.73 & 27.17 & 224.57 & 1030.53 & 1.91 & 830.53 & 2.36 \\
\hline & & 100 & 567.35 & 416.07 & 14.89 & 207.79 & 676.39 & 0.03 & 543.06 & 0.04 \\
\hline & \multirow[t]{2}{*}{0.55} & 200 & 567.35 & 416.07 & 14.89 & 207.79 & 676.44 & 0.02 & 543.11 & 0.03 \\
\hline & & 500 & 629.99 & 413.00 & 14.89 & 207.44 & 676.50 & 0.01 & 543.17 & 0.02 \\
\hline
\end{tabular}

effective unit disposal cost is $c_{\mathrm{d}}+c_{r}$ as given in Eq. (30). In Table 2, we vary $c_{\mathrm{d}}$ as a fraction of $c_{r}$ while maintaining the unit effective disposal cost. We observe that as $c_{\mathrm{d}}$ increases, $\Delta \%$ also increases. This implies that for systems with the same effective unit disposal cost, the savings under the proposed policy are larger when $c_{\mathrm{d}}$ gets larger with respect to $c_{r}$. This interesting result arises from the fact that the constant term $c_{r} \mu_{\mathrm{D}}$ constitutes a larger fraction of the total cost rate under the proposed policy than under the $(Q, r)$ policy. We also observe that $\Delta \%$ decreases as the desired service level $\alpha$ increases. As the fixed ordering cost $K_{\mathrm{o}}$ increases, $\Delta \%$ also decreases (see Table 4). This is largely due to the fact that replenishment costs start constituting a larger portion of the total costs; also, effective costs associated with disposed quantities become larger. The impact of the lead time duration on $\Delta \%$ does not exhibit a clearly distinguishable pattern (see Table 5).

The savings one gets from the disposal option depend on the mean and variance of the net demand. For the same $\sigma$, the performances of the two policies converge ( $\Delta \%$ approaches zero) as the magnitude of net demand $(|\mu|)$ increases. This is because the inventory level, as a stochastic process with negative drift, does not frequently visit the states in the positive half-plane and, thereby, the system does not incur too much inventory holding cost. Hence, no need arises to reduce inventory costs through disposing of some stock. The same argument holds for decreasing $\sigma$ as $\mu$ is kept constant, as well. We observe that changes in the mean net demand rate have more influence on $\Delta \%$ than those in $\sigma$.

In line with these general observations, we find the following. As $\gamma$ decreases, the net demand variability $\sigma$ decreases and, thereby, $\Delta \%$ also decreases. On the other hand, as $\mathrm{CV}_{\mathrm{R}}$ begins to increase, $\Delta \%$ begins to increase. This happens because high variability in return flows leads to accumulation of stock and necessitates the disposal of the excess. Although the effects are more pronounced for high return ratios, we observe, for 
Table 3

Sensitivity analysis $\left(L=0.1, \mu=-200, K_{\mathrm{o}}=1000, \mathrm{CV}_{\mathrm{R}}=1, c_{\mathrm{d}}=1\right.$ and $\left.c_{r}=9\right)$

\begin{tabular}{|c|c|c|c|c|c|c|c|c|}
\hline$\alpha$ & $\gamma$ & $K_{\mathrm{d}}$ & $S^{*}$ & $s^{*}$ & $r^{*}$ & $Q^{*}$ & $\mathrm{TC}^{*}$ & $\Delta \%$ \\
\hline \multirow[t]{9}{*}{0.95} & $0.05 * K_{\mathrm{o}}$ & 7879.92 & 6853.91 & 0.00 & 616.58 & 23357.17 & 14.11 & \\
\hline & 0.90 & $0.1 * K_{\mathrm{o}}$ & 8025.77 & 6733.05 & 0.00 & 615.71 & 23363.93 & 14.09 \\
\hline & & $0.25 * K_{\mathrm{o}}$ & 8285.13 & 6530.34 & 0.00 & 613.74 & 23379.24 & 14.03 \\
\hline & & $0.05 * K_{\mathrm{o}}$ & 5611.26 & 4854.40 & 0.00 & 630.72 & 15219.61 & 5.47 \\
\hline & 0.85 & $0.1 * K_{\mathrm{o}}$ & 5721.10 & 4767.68 & 0.00 & 630.08 & 15223.42 & 5.45 \\
\hline & & $0.25 * K_{\mathrm{o}}$ & 5918.09 & 4623.19 & 0.00 & 628.63 & 15232.01 & 5.40 \\
\hline & & $0.05 * K_{\mathrm{o}}$ & 4505.77 & 3838.95 & 0.00 & 620.91 & 11171.45 & 1.83 \\
\hline & 0.80 & $0.1 * K_{\mathrm{o}}$ & 4579.60 & 3819.73 & 0.00 & 620.43 & 11173.29 & 1.81 \\
\hline & & $0.25 * K_{\mathrm{o}}$ & 4721.44 & 3736.51 & 0.00 & 619.39 & 11177.53 & 1.78 \\
\hline \multirow[t]{9}{*}{0.999} & $0.05 * K_{\mathrm{o}}$ & 9046.76 & 8020.68 & 893.56 & 889.82 & 24286.82 & 13.76 & \\
\hline & 0.90 & $0.1 * K_{\mathrm{o}}$ & 9192.57 & 7899.77 & 893.45 & 889.02 & 24293.53 & 13.74 \\
\hline & & $0.25 * K_{\mathrm{o}}$ & 9451.79 & 7697.01 & 893.18 & 887.23 & 24308.71 & 13.68 \\
\hline & & $0.05 * K_{\mathrm{o}}$ & 6322.22 & 5565.31 & 542.01 & 799.65 & 15776.48 & 5.21 \\
\hline & 0.85 & $0.1 * K_{\mathrm{o}}$ & 6432.16 & 5478.37 & 541.91 & 799.04 & 15780.21 & 5.18 \\
\hline & & $0.25 * K_{\mathrm{o}}$ & 6628.80 & 5333.88 & 541.69 & 797.66 & 15788.62 & 5.13 \\
\hline & & $0.05 * K_{\mathrm{o}}$ & 4988.37 & 4385.24 & 380.43 & 739.28 & 11559.24 & 1.72 \\
\hline & 0.80 & $0.1 * K_{\mathrm{o}}$ & 5127.02 & 4292.19 & 380.27 & 738.81 & 11561.13 & 1.71 \\
\hline & & $0.25 * K_{\mathrm{o}}$ & 5255.22 & 4184.89 & 380.26 & 737.81 & 11565.19 & 1.67 \\
\hline
\end{tabular}

Table 4

Policy comparison $\left(L=0.1, \mu=-20, \mathrm{CV}_{\mathrm{R}}=1, c_{\mathrm{d}}=1\right.$ and $\left.c_{r}=4\right)$

\begin{tabular}{|c|c|c|c|c|c|c|c|c|c|}
\hline \multirow[b]{3}{*}{$\underline{\gamma}$} & \multirow[b]{3}{*}{$K_{\mathrm{d}}$} & \multicolumn{4}{|l|}{$\alpha=0.95$} & \multicolumn{4}{|l|}{$\alpha=0.999$} \\
\hline & & \multicolumn{2}{|c|}{$K_{\mathrm{o}}=500$} & \multicolumn{2}{|c|}{$K_{\mathrm{o}}=1000$} & \multicolumn{2}{|l|}{$K_{\mathrm{o}}=500$} & \multicolumn{2}{|c|}{$K_{\mathrm{o}}=1000$} \\
\hline & & $\mathrm{TC}^{*}$ & $\Delta \%$ & $\mathrm{TC}^{*}$ & $\Delta \%$ & $\mathrm{TC}^{*}$ & $\Delta \%$ & $\mathrm{TC}^{*}$ & $\Delta \%$ \\
\hline \multirow[t]{3}{*}{0.95} & 50 & 2703.28 & 52.01 & 2834.07 & 50.49 & 2870.33 & 50.38 & 2980.15 & 48.95 \\
\hline & 100 & 2710.80 & 51.88 & 2845.26 & 50.29 & 2877.68 & 50.25 & 2991.06 & 48.76 \\
\hline & 250 & 2727.61 & 51.58 & 2870.06 & 49.86 & 2894.11 & 49.97 & 3015.25 & 48.35 \\
\hline \multirow[t]{3}{*}{0.90} & 50 & 1368.60 & 25.06 & 1457.00 & 22.87 & 1439.90 & 23.77 & 1518.34 & 21.79 \\
\hline & 100 & 1372.92 & 24.83 & 1463.14 & 22.54 & 1444.03 & 23.55 & 1524.95 & 21.45 \\
\hline & 250 & 1382.43 & 24.31 & 1476.46 & 21.84 & 1455.14 & 22.96 & 1537.72 & 20.79 \\
\hline \multirow[t]{3}{*}{0.85} & 50 & 915.65 & 11.00 & 988.09 & 9.26 & 962.69 & 9.91 & 1039.61 & 7.39 \\
\hline & 100 & 918.18 & 10.75 & 991.50 & 8.95 & 966.80 & 9.53 & 1048.43 & 6.60 \\
\hline & 250 & 923.62 & 10.23 & 998.64 & 8.29 & 970.04 & 9.22 & 1061.72 & 5.42 \\
\hline \multirow[t]{3}{*}{0.80} & 50 & 688.81 & 4.26 & 753.55 & 3.24 & 718.58 & 3.94 & 780.92 & 2.76 \\
\hline & 100 & 690.18 & 4.07 & 755.26 & 3.02 & 719.76 & 3.79 & 782.75 & 2.53 \\
\hline & 250 & 693.02 & 3.67 & 758.65 & 2.58 & 723.05 & 3.35 & 788.15 & 1.86 \\
\hline \multirow[t]{3}{*}{0.75} & 50 & 554.67 & 1.38 & 615.66 & 0.93 & 577.01 & 1.27 & 634.65 & 0.86 \\
\hline & 100 & 555.30 & 1.27 & 616.38 & 0.81 & 577.64 & 1.16 & 635.36 & 0.75 \\
\hline & 250 & 556.54 & 1.05 & 617.66 & 0.60 & 578.80 & 0.97 & 637.03 & 0.49 \\
\hline \multirow[t]{3}{*}{0.70} & 50 & 468.04 & 0.35 & 527.44 & 0.20 & 485.78 & 0.31 & 542.66 & 0.16 \\
\hline & 100 & 468.28 & 0.30 & 527.67 & 0.16 & 485.96 & 0.27 & 542.72 & 0.15 \\
\hline & 468.68 & 0.21 & 528.02 & 0.09 & 486.32 & 0.20 & 543.06 & 0.09 & \\
\hline
\end{tabular}

instance in Fig. 10, that the disposal policy results in substantial cost savings even for the systems with moderate return ratios but high return variability (e.g. when $C_{\mathrm{R}}=2$ and $\gamma=0.75$ the cost rate difference is approximately $10 \%$ ). For the same $\mathrm{CV}_{\mathrm{R}}, \Delta \%$ increases as $|\mu|$ increases for slow-moving to moderate demands. For example, in the cases considered in Fig. 9 and Fig. 10, we see that for $\mathrm{CV}_{\mathrm{R}}=0.5,1,1.5,2, \alpha=0.99$ and $\gamma=0.90$, the cost rate difference takes on the range of values, $0.91-36.76$ and $5.29-54.71$ for $\mu=-1$ and -20 , resp. (Recall that we set $C V_{D}=0.3$ throughout the numerical study.) We see that the increase in $\Delta \%$ is 
Table 5

Policy comparison $\left(\mu=-1, K_{\mathrm{o}}=500, \mathrm{CV}_{\mathrm{R}}=1, c_{\mathrm{d}}=1\right.$ and $\left.c_{r}=4\right)$

\begin{tabular}{|c|c|c|c|c|c|c|c|c|c|}
\hline \multirow[b]{3}{*}{$\gamma$} & \multirow[b]{3}{*}{$K_{\mathrm{d}}$} & \multicolumn{4}{|c|}{$\alpha=0.95$} & \multicolumn{4}{|c|}{$\alpha=0.999$} \\
\hline & & \multicolumn{2}{|l|}{$L=0.1$} & \multicolumn{2}{|l|}{$L=1$} & \multicolumn{2}{|l|}{$L=0.1$} & \multicolumn{2}{|l|}{$L=1$} \\
\hline & & $\mathrm{TC}^{*}$ & $\Delta \%$ & $\mathrm{TC}^{*}$ & $\Delta \%$ & $\mathrm{TC}^{*}$ & $\Delta \%$ & $\mathrm{TC}^{*}$ & $\Delta \%$ \\
\hline \multirow[t]{3}{*}{0.95} & 50 & 183.49 & 40.69 & 178.86 & 40.83 & 187.39 & 39.75 & 210.34 & 36.42 \\
\hline & 100 & 185.22 & 40.13 & 180.56 & 40.27 & 189.39 & 39.11 & 211.96 & 35.93 \\
\hline & 250 & 188.88 & 38.95 & 184.15 & 39.08 & 193.19 & 37.88 & 215.39 & 34.89 \\
\hline \multirow[t]{3}{*}{0.90} & 50 & 101.80 & 12.53 & 99.79 & 12.66 & 104.33 & 10.99 & 114.33 & 10.55 \\
\hline & 100 & 102.54 & 11.89 & 100.53 & 12.01 & 104.87 & 10.52 & 114.88 & 10.12 \\
\hline & 250 & 104.02 & 10.62 & 102.00 & 10.73 & 106.02 & 9.55 & 116.33 & 8.99 \\
\hline \multirow[t]{3}{*}{0.85} & 50 & 73.82 & 3.12 & 72.46 & 3.17 & 74.42 & 3.02 & 81.41 & 2.69 \\
\hline & 100 & 74.11 & 2.73 & 72.75 & 2.77 & 74.71 & 2.64 & 81.70 & 2.36 \\
\hline & 250 & 74.63 & 2.04 & 73.27 & 2.08 & 75.21 & 1.98 & 82.19 & 1.77 \\
\hline \multirow[t]{3}{*}{0.80} & 50 & 60.25 & 0.61 & 59.13 & 0.63 & 60.85 & 0.28 & 65.78 & 0.33 \\
\hline & 100 & 60.34 & 0.46 & 59.22 & 0.47 & 60.91 & 0.18 & 65.85 & 0.23 \\
\hline & 250 & 60.47 & 0.25 & 59.35 & 0.25 & 61.00 & 0.04 & 65.91 & 0.13 \\
\hline \multirow[t]{3}{*}{0.75} & 50 & 52.73 & 0.00 & 51.71 & 0.00 & 53.05 & 0.00 & 56.80 & 0.00 \\
\hline & 100 & 52.73 & 0.00 & 51.71 & 0.00 & 53.05 & 0.00 & 56.80 & 0.00 \\
\hline & 250 & 52.73 & 0.00 & 51.71 & 0.00 & 53.05 & 0.00 & 56.80 & 0.00 \\
\hline \multirow[t]{3}{*}{0.70} & 50 & 48.07 & 0.00 & 47.11 & 0.00 & 48.33 & 0.00 & 51.27 & 0.00 \\
\hline & 100 & 48.07 & 0.00 & 47.11 & 0.00 & 48.33 & 0.00 & 51.27 & 0.00 \\
\hline & 250 & 48.07 & 0.00 & 47.11 & 0.00 & 48.33 & 0.00 & 51.27 & 0.00 \\
\hline
\end{tabular}

more pronounced for $\mu=-20$ than for $\mu=-1$. However, as $|\mu|$ gets larger, we observe that $\Delta \%$ drops to zero (for $\mu=-200$ and $\mu=-2000$ ), which follows from the interplay between $|\mu|$ and $\sigma$ as mentioned above.

Previously, Teunter and Vlachos [19] have shown that, for zero fixed disposal cost, disposals are beneficial only when the item is slow-moving and the return rate is high. Our results support this finding. This is as expected since addition of a fixed disposal cost makes disposal decisions less desirable. However, we should also report our observation that systems with high actual demand rates may also experience savings if the net demand rate for the system is low. For instance, with $\gamma=0.95, \mu=-200$ and $c_{r}=9$ in Table 3, the actual demand rate is 4000 units per year with an annual cost of capital of slightly more than $11 \%$ and, the corresponding saving is $14.1 \%$. Hence, the actual demand figures may be misleading in stating a priori when disposals may be beneficial. We recommend that the net demand's mean and variability be considered. We also observe that for large net demand values, the fixed ordering and disposal costs must also be relatively

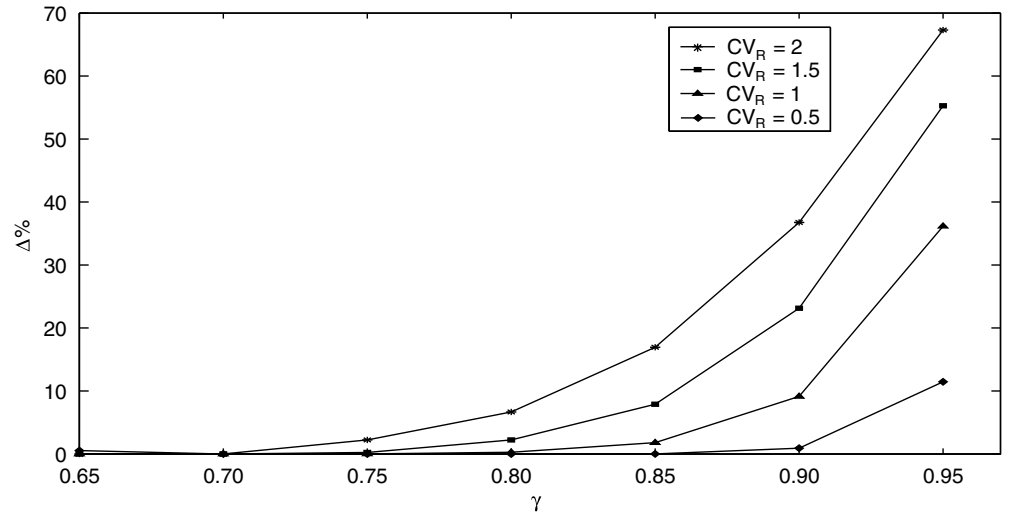

Fig. 9. Disposal vs. no disposal $\left(\mu=-1, L=0.1, \alpha=0.99, K_{\mathrm{o}}=1000, K_{\mathrm{d}}=100, c_{\mathrm{d}}=1, c_{r}=4\right)$. 


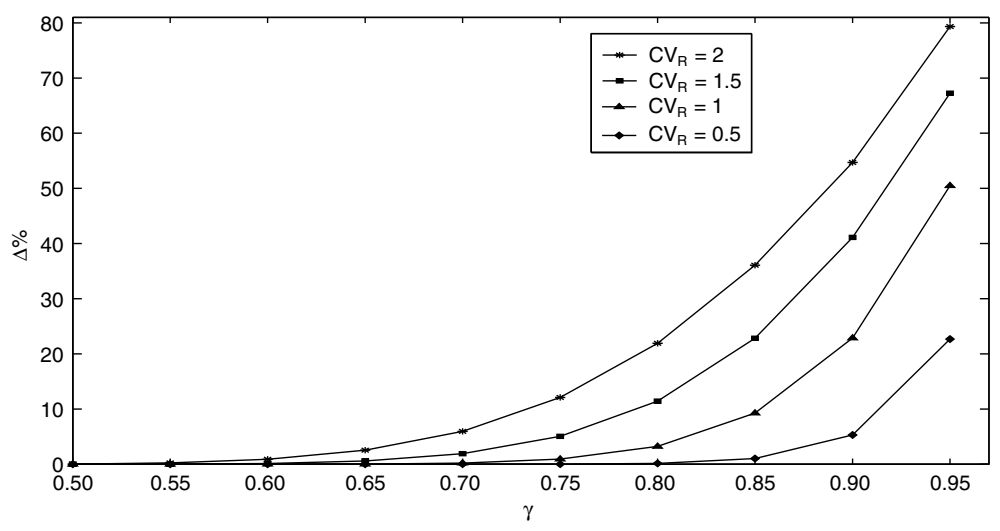

Fig. 10. Disposal vs. no disposal $\left(\mu=-20, L=0.1, \alpha=0.99, K_{\mathrm{o}}=1000, K_{\mathrm{d}}=100, c_{\mathrm{d}}=1, c_{r}=4\right)$.

high to obtain benefits from disposals. For instance, for $\mu=-20,-200$ and -2000 , equivalent cost savings $(\approx 14 \%)$ were obtained only if the ratios of fixed costs to demand rates $\left(K_{\mathrm{o}} /|\mu|, K_{\mathrm{d}} /|\mu|\right)$ were maintained as $|\mu|$ grows large for the same $\mathrm{CV}$; otherwise, disposal option becomes uneconomical quickly as $|\mu|$ grows large. The above behavior is seen at all service levels considered.

In addition to the tabulated results, we should report summaries of our findings on policy comparison over the entire experimental set for negative drift: Over all experiment instances with negative drift (24588 out of a total of 24,912 instances), we have observed $4 \%$ to a have a mean value of $4.5 \%$ and a maximum of $76.4 \%$. We see that incorporating the disposal option in the presence of positive fixed disposal costs can result in savings. The savings diminish for large net demand rates, 1 ow net demand variance, low fixed ordering/disposal costs and low holding costs.

\section{Conclusion}

In this paper, we considered a single-location inventory system for a reusable item with stochastic external demand and an autonomous supply providing an independent inflow of material in addition to a regular supplier with constant delivery lead time. Our model differs from the available models on resuables facing random demand in that we allow for non-zero fixed disposal costs. We proposed a continuous review, four parameter replenishment-disposal policy for inventory control. As such, we are able to determine the optimal disposal batch size as well as the disposal action trigger level, reordering quantity and its timing. We modeled the net demand process as the superposition of the normally distributed demand and inflows resulting in inventory levels represented by a Brownian motion. Under the assumption of at most one order outstanding at any time, the operating characteristics of the system were derived and the optimal values of the policy parameters that minimize expected total cost rate were obtained under a fill rate measure constraint.

The benefits of a disposal option on reduction of cost have been studied in the literature for systems with random demand when fixed disposal cost is zero. The main conclusion from that research has been that disposal option can lead to considerable savings, but only for cases with slow-moving items and large return ratios. In our study, we strengthen these findings and extend them in a number of directions. First, disposals of excess stock may result in significant savings even in the presence of non-zero fixed disposal costs. Secondly, we observe in our numerical study that benefits arising from disposals depend on the net demand rate and that savings are possible for large actual demand rates if the return rates are also high. Substantial savings can be achieved even for moderate return rates as long as the net demand variability and fixed costs are sufficiently high.

There are a number of possible extensions to our model. Non-linear holding costs may be incorporated which would imply further penalization of excessive inventory build-up and increase the attractiveness of disposals. Also the assumption of constant lead times may be relaxed to allow for uncertainty in delivery times. In this study, we considered an independent inflow; correlated demand and return processes would be 
interesting to study as well. Finally, another possible extension that can build on the elements of our model is to look at the same problem with an NPV approach instead of a cost rate. Such an approach would be most useful to study the impact of additional uncertainties in the operating environment such as prices and discount rates.

\section{Appendix}

\section{Proof of Proposition 3.2}

(i) Let $\pi_{+}(x)=P_{x, a}^{b}$. Then $\pi_{+}(x)$ satisfies the following differential equation (see [8, p. 231]):

$\frac{1}{2} \sigma^{2} \frac{\mathrm{d}^{2} \pi_{+}(x)}{\mathrm{d} x^{2}}+\mu \frac{\mathrm{d} \pi_{+}(x)}{\mathrm{d} x}=0$.

The general solution of (31) is given by $\pi_{+}(x)=A+B \mathrm{e}^{-\frac{2 \mu x}{\sigma^{2}}}$, and solving with the boundary conditions $\pi_{+}(a)=1$ and $\pi_{+}(b)=0$ yields the following first hitting probability

$\pi_{+}(x)=\frac{\left(\mathrm{e}^{-\frac{2 \mu x}{\sigma^{2}}}-\mathrm{e}^{-\frac{2 \mu b}{\sigma^{2}}}\right)}{\left(\mathrm{e}^{-\frac{2 \mu a}{\sigma^{2}}}-\mathrm{e}^{-\frac{2 \mu b}{\sigma^{2}}}\right)}$

The expression for $P_{x, b}^{a}$ immediately follows from setting $P_{x, b}^{a}=1-P_{x, a}^{b}$.

(ii) The moment generating function, $\gamma(x)$, of the first escape time from the interval $[b, a], T_{x, b a}$, satisfies the following differential equation (see $[8$, p. 230]):

$\frac{1}{2} \sigma^{2} \frac{\mathrm{d}^{2} \gamma(x)}{\mathrm{d} x^{2}}+\mu \frac{\mathrm{d} \gamma(x)}{\mathrm{d} x}=s \gamma(x)$

with the boundary condition $\gamma(b)=\gamma(a)=1$. The general solution of (32) is given by

$\gamma(x)=A \mathrm{e}^{x \theta_{1}(s)}+B \mathrm{e}^{x \theta_{2}(s)}$,

where

$\theta_{1}(s)=\frac{-\mu-\sqrt{\mu^{2}+2 s \sigma^{2}}}{\sigma^{2}} \quad \theta_{2}(s)=\frac{-\mu+\sqrt{\mu^{2}+2 s \sigma^{2}}}{\sigma^{2}}$

Solving (33) with the boundary condition, we have

$\gamma(x)=\left[\frac{\mathrm{e}^{b \theta_{1}(s)+a \theta_{2}(s)}-\mathrm{e}^{b\left[\theta_{1}(s)+\theta_{2}(s)\right]}}{\mathrm{e}^{2 b \theta_{1}(s)+a \theta_{2}(s)}-\mathrm{e}^{a \theta_{1}(s)+b\left[\theta_{1}(s)+\theta_{2}(s)\right]}}\right] \mathrm{e}^{x \theta_{1}(s)}+\left[\frac{\mathrm{e}^{b \theta_{1}(s)}-\mathrm{e}^{a \theta_{1}(s)}}{\mathrm{e}^{b \theta_{1}(s)+a \theta_{2}(s)}-\mathrm{e}^{b \theta_{2}(s)+a \theta_{1}(s)}}\right] \mathrm{e}^{x \theta_{2}(s)}$.

Hence,

$$
E\left(T_{x, b a}\right)=-\left.\gamma^{\prime}(x)\right|_{s=0}=-\frac{x}{\mu}+\frac{(a-b)}{\mu\left(\mathrm{e}^{-\frac{2 \mu a}{\sigma^{2}}}-\mathrm{e}^{-\frac{2 \mu b}{\sigma^{2}}}\right)} \mathrm{e}^{-\frac{2 \mu x}{\sigma^{2}}}+\frac{b \mathrm{e}^{-\frac{2 \mu a}{\sigma^{2}}}-a \mathrm{e}^{-\frac{2 \mu b}{\sigma^{2}}}}{\mu\left(\mathrm{e}^{-\frac{2 \mu a}{\sigma^{2}}}-\mathrm{e}^{-\frac{2 \mu b}{\sigma^{2}}}\right)}
$$

\section{Proof of Proposition 3.3}

Using the probability density function given by Proposition 3.1 we can find the expected inventory carried until the process escapes from strip $[b, a]$ as follows:

$$
\begin{aligned}
& A_{x, b a}=\int_{0}^{\infty} \int_{b}^{a} y f_{X(t)}(y, t, x) \mathrm{d} y \mathrm{~d} t \\
& =\int_{0}^{\infty} \int_{b}^{a} y \mathrm{e}^{\frac{\mu(y-x)}{\sigma^{2}}} \sum_{n=1}^{\infty} a_{n} \mathrm{e}^{-\lambda_{n} t} \sin \left(\frac{n \pi(y-b)}{a-b}\right) \mathrm{d} y \mathrm{~d} t \\
& =\sum_{n=1}^{\infty} a_{n} \mathrm{e}^{-\frac{\mu x}{\sigma^{2}}} \int_{0}^{\infty} \mathrm{e}^{-\lambda_{n} t} F(b, a, n ; \mu) \mathrm{d} t=\sum_{n=1}^{\infty} \frac{a_{n}}{\lambda_{n}} \mathrm{e}^{-\frac{\mu x}{\sigma^{2}}} F(b, a, n ; \mu),
\end{aligned}
$$


where

$$
\begin{aligned}
& F(b, a, n ; \mu)=\int_{b}^{a} y \mathrm{e}^{\frac{\mu y}{\sigma^{2}}} \sin \left(\frac{n \pi(y-b)}{a-b}\right) \mathrm{d} y \\
& =y\left[\frac{(a-b) \mathrm{e}^{\frac{\mu y}{\sigma^{2}}}}{n \pi\left[1+\left(\frac{\mu(a-b)}{\sigma^{2} n \pi}\right)^{2}\right]}\left[\frac{\mu(a-b)}{\sigma^{2} n \pi} \sin \left(\frac{n \pi(y-b)}{a-b}\right)-\cos \left(\frac{n \pi(y-b)}{a-b}\right)\right]\right] \\
& \quad-\int_{b}^{a} \frac{(a-b) \mathrm{e}^{\frac{\mu \nu}{\sigma^{2}}}}{n \pi\left[1+\left(\frac{\mu(a-b)}{\sigma^{2} n \pi}\right)^{2}\right]}\left[\frac{\mu(a-b)}{\sigma^{2} n \pi} \sin \left(\frac{n \pi(y-b)}{a-b}\right)-\cos \left(\frac{n \pi(y-b)}{a-b}\right)\right] \mathrm{d} y .
\end{aligned}
$$

\section{Letting}

$$
\begin{aligned}
& K_{1}=\frac{(a-b)}{n \pi\left(1+K_{2}^{2}\right)}, \\
& K_{2}=\frac{\mu(a-b)}{\sigma^{2} n \pi}
\end{aligned}
$$

and observing that $n$ takes integer values with $\sin (n \pi)=0$, we have

$$
\begin{aligned}
F(b, a, n ; \mu) & =a K_{1} \mathrm{e}^{\frac{\mu a}{\sigma^{2}}}\left[K_{2} \sin (n \pi)-\cos (n \pi)\right]+b K_{1} \mathrm{e}^{\frac{\mu b}{\sigma^{2}}}+2 K_{1}^{2} K_{2}\left[\mathrm{e}^{\frac{\mu a}{\sigma^{2}}} \cos (n \pi)-\mathrm{e}^{\frac{\mu b}{\sigma^{2}}}\right] \\
& =-K_{1}\left[a \mathrm{e}^{\frac{\mu a}{\sigma^{2}}} \cos (n \pi)-b \mathrm{e}^{\frac{\mu b}{\sigma^{2}}}\right]+2 K_{1}^{2} K_{2}\left[\mathrm{e}^{\frac{\mu a}{\sigma^{2}}} \cos (n \pi)-\mathrm{e}^{\frac{\mu b}{\sigma^{2}}}\right] .
\end{aligned}
$$

Proof of Eq. (19)

From Proposition 3.2 (i) we know

$$
P_{x, S}^{r}=\frac{\mathrm{e}^{-\frac{2 \mu x}{\sigma^{2}}}-\mathrm{e}^{-\frac{2 \mu r}{\sigma^{2}}}}{\mathrm{e}^{-\frac{2 \mu S}{\sigma^{2}}}-\mathrm{e}^{-\frac{2 \mu r}{\sigma^{2}}}}, P_{s, S}^{r}=\frac{\mathrm{e}^{-\frac{2 \mu s}{\sigma^{2}}}-\mathrm{e}^{-\frac{2 \mu r}{\sigma^{2}}}}{\mathrm{e}^{-\frac{2 \mu S}{\sigma^{2}}}-\mathrm{e}^{-\frac{2 \mu \nu}{\sigma^{2}}}}, P_{s, r}^{S}=\frac{\mathrm{e}^{-\frac{2 \mu S}{\sigma^{2}}}-\mathrm{e}^{-\frac{2 \mu s}{\sigma^{2}}}}{\mathrm{e}^{-\frac{2 \mu S}{\sigma^{2}}}-\mathrm{e}^{-\frac{2 \mu r}{\sigma^{2}}}} .
$$

Hence, we can write the conditional expectation as

$$
E\left(N_{x}\right)=\sum_{n=1}^{\infty} n P_{x, S}^{r}\left(P_{s, S}^{r}\right)^{(n-1)} P_{s, r}^{S}=P_{x, S}^{r} P_{s, r}^{S}\left[1+2 P_{s, S}^{r}+3\left(P_{s, S}^{r}\right)^{2}+\cdots\right]=\frac{\mathrm{e}^{-\frac{2 \mu x}{\sigma^{2}}}-\mathrm{e}^{-\frac{2 \mu r}{\sigma^{2}}}}{\mathrm{e}^{-\frac{2 \mu S}{\sigma^{2}}}-\mathrm{e}^{-\frac{2 \mu s}{\sigma^{2}}}} .
$$

\section{References}

[1] K. Auguston, Reduce packaging cost with reusable containers, Modern Materials Handling 47 (8) (1992) $42-45$.

[2] J.L. Beltran, D. Krass, Dynamic lot sizing with returning items and disposals, IIE Transactions 34 (5) (2002) $437-448$.

[3] M.B. Berthelsen, Production Manager, Supply Chain-Tuborg-Fredericia Brewery. Personal Communication, 24.08.2005.

[4] CWC and Skookum Inc., A Model for a Bottlewashing Plant, Report No. GL-97-12.

[5] City of Newark Fire Prevention Bureau, General Provisions for Outside Storage of Wooden Pallets, Information Letter No. 8.

[6] G.M. Constantinides, Stochastic cash management with fixed and proportional costs, Management Science 22 (12) (1976) 1320-1331.

[7] G.M. Constantinides, S.F. Richard, Existence of optimal simple policies for discounted-cost inventory and cash management in continuous time, Operations Research 26 (4) (1978) 620-636.

[8] D.R. Cox, H.D. Miller, The Theory of Stochastic Processes, Chapman and Hall, New York, 1977.

[9] R. Dekker and E. van der Laan, Inventory Control in Reverse Logistics, in: Guide (Eds.), Business Aspects of Closed-Loop Supply Chains, 2003, pp. 175-203.

[10] European Parliament and Council Directive 94/62/EC of 20 December 1994 on packaging and packaging waste, Official Journal, L $365,10-23,31 / 12 / 1994$.

[11] M. Fleischmann, R. Kuik, R. Dekker, Controlling inventories with stochastic item returns: A basic model, European Journal of Operational Research 138 (2002) 63-75.

[12] B. Golany, J. Yang, G. Yu, Economic lot-sizing with remanufacturing options, IIE Transactions 33 (1) (2001) $195-203$. 
[13] P.L. González-Torre, B. Adenso-Díaz, H. Artiba, Environmental and reverse logistics policies in european bottling and packaging firms, International Journal of Production Economics 88 (1) (2004) 95-104.

[14] G.J. Hadley, T.M. Whitin, Analysis of Inventory Systems, Prentice-Hall, Englewood Cliffs, New Jersey, 1963.

[15] D.P. Heyman, Optimal disposal policies for a single-item inventory system with returns, Naval Research Logistics Quarterly 24 (1977) 385-405.

[16] L. Kroon, G. Vrijens, Returnable containers: An example of reverse logistics, International Journal of Physical Distribution \& Logistics Management 25 (2) (1995) 56-68.

[17] B.Platt and D. Rowe, Reduce, Reuse, Refill!, Washington, DC: Institute for Local Self-Reliance, April 2002, produced under a joint project with the GrassRoots Recycling Network. <http://www.grrn.org/beverage/refillables/refill_report.pdf $>$.

[18] S. Ross, Stochastic Processes, Wiley, New York, 1983.

[19] R.H. Teunter, D. Vlachos, On the necessity of a disposal option for returned products that can be remanufactured, International Journal of Production Economics 75 (3) (2002) 257-266.

[20] E. van der Laan, R. Dekker, M. Salomon, A. Ridder, An $(s, Q)$ inventory model with remanufacturing and disposal, International Journal of Production Economics 46-47 (1996) 339-350.

[21] E. van der Laan, M. Salomon, R. Dekker, An investigation of lead-time effects in manufacturing/remanufacturing systems under simple push and pull control strategies, European Journal of Operational Research 115 (1999) 195-214.

[22] E. van der Laan, M. Salomon, Production planning and inventory control with remanufacturing and disposal, European Journal of Operational Research 102 (1997) 264-278.

[23] S. Vandermerwe, M.D. Oliff, Customers drive corporations green, Long Range Planning 23 (6) (1990) $10-16$.

[24] C.E. Witt, Returnable distribution packaging saves money, Material Handling Engineering 48 (2) (1993) 14. 